INRA Prod. Anim., 1999, 12 (1), 11-28

\section{B. LEBRET, L. LEFAUCHEUR, J. MOUROT}

INRA Stati on de Recherches Porcines, 35590 Saint-Gilles

lebret@st-gilles.rennes.inra.fr

\title{
La qualité de la viande de porc Influence des facteurs d'élevage non génétiques sur les caractéristiques du tissu musculaire
}

La qualité de la fraction maigre de la viande de porc est fonction du type génétique, des conditions d'abattage et des conditions d'élevage des animaux. Les influences néfastes de certaines anomalies génétiques et de mauvaises conditions d'abattage sur la qualité sont relativement bien connues et maîtrisables au niveau de la production. Dès lors que ces défauts majeurs sont écartés, l'amélioration de la qualité des viandes nécessite de prendre en considération l'influence des facteurs d'élevage non génétiques sur les caractéristiques du tissu maigre, en relation avec la qualité des produits.

Les effets du type génétique et des conditions d'abattage des porcs sur la qualité de la viande sont maintenant relativement bien connus et ont déià fait l'objet de plusieurs articles de synthese (Monin 1983 et 1988, Sellier 1988 et 1998, Sellier et Monin 1994). Cependant, la qualité de la viande peut égale-

\section{Résumé}

Les interactions entre le type génétique, les conditions d'élevage et les conditions d'abattage des animaux déterminent la qualité de la fraction maigre des viandes de porc. L'influence du type génétique, en particulier la présence des gènes majeurs $\mathrm{Hal}$ et $\mathrm{RN}$-, ainsi que les conditions d'abattage ont des conséquences importantes et maintenant bien établies sur la qualité. Une fois ces facteurs maîtrisés, l'amélioration de la qualité des viandes nécessite la prise en compte de l'influence des facteurs d'élevage non génétiques sur les propriétés des tissus maigres, ce qui constitue l'objectif de la présente revue. Cet article présente les caractéristiques du tissu musculaire, en particulier les fibres musculaires, les lipides intramusculaires et le tissu conjonctif, puis les relations entre ces caractéristiques et les composantes organoleptique, technologique, nutritionnelle et hygiénique de la qualité. Nous décrivons ensuite les effets de différents facteurs d'élevage : âge et poids à l'abattage, type sexuel, niveau et nature de la ration alimentaire, promoteurs de croissance, température ambiante, activité physique et système d'élevage, sur les propriétés des muscles et leur incidence sur la qualité des viandes. Si certaines relations sont bien établies, comme l'effet favorable, jusqu'à $3 \%$ environ, du taux de lipides intramusculaires sur la qualité sensorielle, l'influence des propriétés des fibres (type contractile et métabolique, diamètre) sur la qualité des viandes est moins claire. La détermination des caractéristiques musculaires favorables aux différentes composantes de la qualité, ainsi qu'une meilleure connaissance des mécanismes de différenciation et de développement des tissus conduisant à ces caractéristiques sont nécessaires, afin de pouvoir répondre aux besoins variés des transformateurs et consommateurs. ment être influencée par les conditions d'élevage des animaux : l'âge et le poids au stade d'abattage, le type sexuel, le niveau de la ration alimentaire ainsi que l'équilibre entre les principaux nutriments, les apports vitaminiques, l'administration de promoteurs de croissance (pST, bêta-agonistes), la température ambiante, l'activité physique et le système d'élevage.

Après avoir passé en revue l'influence de ces différents facteurs sur la qualité du tissu adipeux chez le porc (Lebret et Mourot 1998), nous nous intéressons ici à leurs effets sur la qualité du tissu musculaire. Le présent article décrit les caractéristiques du tissu musculaire (ou tissu maigre de la viande) et leurs relations avec les différentes composantes de la qualité (organoleptique, technologique, nutritionnelle, hygiénique), puis présente l'influence des facteurs d'élevage sur les caractéristiques qualitatives du tissu maigre.

\section{1 / Caractéristiques du tissu musculaire}

\section{1 / A l'abattage}

Le muscle squelettique est quantitativement le tissu le plus important de l'organisme. Au poids commercial a l'abattage des porcs, environ $105 \mathrm{~kg}$ de poids vif, ce tissu représente jusqu'à $60 \%$ du poids de la carcasse et 40 à 
$45 \%$ des protéines totales de l'individu. II est majoritairement composé d'eau (environ $73 \%$ ) et contient environ $20 \%$ de protéines, 1 à $6 \%$ de lipides et 1 à $2 \%$ de glucides et minéraux. Au plan structural, le muscle est un tissu composite qui renferme, outre les fibres musculaires, des tissus conjonctif, adipeux, vasculaire et nerveux. Les vaisseaux sanguins, les nerfs et les cellules adipeuses intramusculaires sont localisés au niveau de la trame conjonctive, autour des fibres et des faisceaux de fibres musculaires.

\section{a / Les fibres musculaires}

Les fibres musculaires diffèrent par leurs propriétés morphologiques, biochimiques et physiologiques (Cassens et Cooper 1971). La conjugaison des caractéristiques contractiles (vitesse de contraction lente ou rapide) et métaboliques (métabolisme oxydatif, oxydoglycolytique ou glycolytique) permet de classer les fibres en types $\beta R$ (lent rouge), $\alpha R$ (rapide rouge) et aW (rapide blanc) par histoenzymologie (Ashmore et Doerr 1971 : tableau 1). Chez le porc, les fibres musculaires sont typiquement disposées en îlots de fibres bR entourées par une première couronne de fibres $a R$ et une seconde, plus externe, de fibres aW (Lefaucheur et Vigneron 1986).

La régénération de I'ATP nécessaire à la contraction des fibres musculaires est assurée par deux voies métaboliques principales. La voie glycolytique transforme le glycogène en acide lactique via la glycolyse, sans consommation d'oxygène. La voie oxydative produit de l'énergie a partir du glycogène, du glucose, des acides gras et de certains acides aminés, en consommant de l'oxygène. L'ATP peut également être régénéré à partir de la phosphocréatine, ainsi que par une réaction biochimique faisant intervenir la myokinase. Dans un muscle, la contribution relative des voies oxydatives et glycolytiques à la production d'énergie peut être évaluée en mesurant le potentiel d'activité d'enzymes spécifiques de chacune de ces deux voies : la lactate déshydrogénase (LDH) pour le métabolisme glycolytique, la citrate synthase (CS), la succinodéshydrogénase (SDH) ou l'isocitrate déshydrogénase (ICDH) pour la voie oxydative et la B-hydroxyacyl coenzyme $A$ déshydrogénase (HAD) pour la ß-oxydation des acides gras.

\section{b / Les lipides intramusculaires}

Chez le porc, la teneur en lipides intramusculaires varie entre 1 et $6 \%$ selon les muscles. Ces lipides sont constitués de lipides de réserve, les triglycérides, et de lipides membranaires, les phospholipides, dont les proportions respectives varient selon les muscles. Ainsi, la différence de teneur en lipides entre les muscles Longi ssimus (muscle blanc) et Semispinalis capitis (muscle rouge) est essentiellement liée à une teneur différente en triglycérides ( 1,0 et 5,0\% respectivement), la teneur en phospholipides étant légèrement supérieure dans le Semi spi nali s (0,67 \% vs 0,54 \%) (Fernandez et al 1995). La teneur plus élevée des muscles rouges en phospholipides est souvent attribuée à un nombre plus élevé de mitochondries.

Au sein du muscle, les triglycérides sont à la fois présents à l'intérieur des fibres musculaires sous forme de gouttelettes lipidiques et à l'extérieur des fibres dans des adipocytes intramusculaires. Ces cellules sont soit isolées entre les fibres musculaires, soit regroupées sous forme de tissu le long des faisceaux de fibres à proximité de vaisseaux sanguins. La proportion des adipocytes isolés représente 10 à $20 \%$ des adipocytes totaux d'un muscle (M.J. Chora et al 1996, non publié).

II a souvent été considéré que la teneur en lipides augmente avec le caractère oxydatif des muscles. Plusieurs études indiquent en effet une teneur en lipides supérieure dans le muscle Semispinalis par rapport au Longi ssi mus (Lefaucheur et al 1991 et 1992). Cependant, à l'inverse, aucune différence de teneur en lipides totaux n'a été trouvée entre les muscles Longissimus et Biceps femoris (Essen-Gustavsson et al 1994), ni entre les muscles Longi ssi mus et Trapezi us ou Masseter, deux muscles oxydatifs (Leseigneur-Meynier et Gandemer 1991). De même, Larzul et al (1997) n'ont observé aucune relation entre la teneur en lipides et la composition en types de fibres du muscle Longi ssi mus. En conséquence, aucune relation générale ne semble exister entre le type métabolique ou contractile d'un muscle et sa teneur en lipides totaux. En revanche, il a été clairement démontré que les fibres oxydatives sont plus riches en lipides que les fibres

Tableau 1. Caractéristiques des fibres musculaires chez le porc.

\begin{tabular}{|l|l|l|l|}
\hline Type contractile & Lent (ß) & \multicolumn{2}{|c|}{ Rapide $(\alpha)$} \\
\hline Type métabolique & Oxydatif & Oxydo-glycolytique & Glycolytique \\
Classification & SR & $\alpha R$ & $\alpha \mathrm{W}$ \\
ATPase myofibrillaire & Faible & Elevée & Elevée \\
Diamètre & Faible & Faible & Elevé \\
Densité capillaire & Elevée & Elevée & Faible \\
Nombre de mitochondries & Elevé & Elevé & Faible \\
Teneur en myoglobine & Elevée & Elevée & Faible \\
Teneur en lipides & Elevée & Moyenne & Faible \\
Teneur en glycogène & Faible & Elevée & Elevée \\
Résistance à la fatigue & Elevée & Moyenne & Faible \\
\hline
\end{tabular}


glycolytiques (Cassens et Cooper 1971, Essen-Gustavsson et al 1994) (tableau 1). Toutefois, les lipides contenus dans les fibres ne représentent qu'une faible proportion des lipides intramusculaires totaux, la majorité des lipides étant localisée dans les adipocytes intramusculaires, ce qui permet d'expliquer l'absence de relation entre le type de fibres et la teneur en lipides totaux d'un muscle.

\section{c / Le glycogène}

Le glycogène intramusculaire, qui représente environ $1 \%$ du poids du muscle, est stocké dans les fibres musculaires sous forme de granules localisés dans le sarcoplasme. Le glycogène constitue la source énergétique principale des fibres glycolytiques (blanches) qui le dégradent rapidement en acide lactique lorsqu'elles se contractent. $E n$ général, les fibres à vitesse de contraction lente contiennent moins de glycogène que les fibres rapides (Cassens et Cooper 1971) (tableau 1), car elles sont beaucoup plus fréquemment sollicitées que les fibres rapides, en particulier rapides glycolytiques.

\section{d / Le tissu conjonctif}

Le tissu conjonctif forme dans le muscle un réseau continu, hiérarchisé en trois niveaux : l'épimysium qui enveloppe le muscle et se poursuit par les tendons, le périmysium qui entoure les faisceaux de fibres musculaires et l'endomysium qui est présent autour de chaque fibre musculaire. Le composant majeur du tissu conjonctif musculaire est le collagène, qui représente 50 à $80 \%$ du poids $\mathrm{sec}$ de la trame conjonctive. Le collagène constitue une famille de protéines qui se différencient par leur structure primaire et leur arrangement spatial. On distingue les collagènes fibrillaires (types I et III) et les collagènes non fibrillaires (type IV) qui forment des réseaux (Bailey et Light 1989). Le muscle renferme essentiellement du collagène de type I ( $95 \%$ du collagène musculaire), présent à tous les niveaux de la trame conjonctive. Le type III est localisé au niveau du périmysium alors que le type IV, spécifique des lames basales, est circonscrit à l'endomysium (Bailey et Light 1989).

\section{2 / Au cours du développement}

\section{a / Les fibres musculaires}

Chez le porc comme chez de nombreux mammifères, le développement des fibres musculaires est biphasique, caractérisé par l'apparition de deux génerations successives de fibres au cours de la période fœtale (Ashmore et al 1973). Autour des fibres de première génération, qui se mettent en place jusqu'au $55^{\mathrm{e}}$ jour de gestation, se mettent en place les fibres de deuxième génération entre le $55^{\mathrm{e}}$ et le $90^{\mathrm{e}}-95^{\mathrm{e}}$ jour de gestation (Wigmore et Stickland 1983). Le nombre total de fibres musculaires étant définitivement fixé avant la naissance dans l'espèce porcine, la croissance musculaire postnatale résulte uniquement d'une hypertrophie des fibres existantes
(Ashmore et al 1973). Le potentiel de croissance musculaire du porc dépend principalement du nombre total de fibres musculaires (Miller et al 1975), ce qui explique l'absence de corrélation entre le diamètre des fibres musculaires et la teneur en muscle d'un animal (Livingston et al 1966).

Concernant l'origine des différents types de fibres musculaires, on a longtemps admis que la totalité des fibres de première génération donnait des fibres lentes, alors que les fibres secondaires évoluaient vers le type lent ou rapide, avec des différences d'intensité selon les muscles (Lefaucheur 1989). Des études immunohistologiques réalisées par Lefaucheur et al (1995) ont confirmé ce schéma de maturation pour la partie rouge (interne) du muscle Semitendinosus, mais indiquent que les myotubes primaires donnent des fibres rapides dans la partie blanche (superficielle) de ce muscle. Le typage des fibres musculaires étant basé sur le polymorphisme des chaînes lourdes de la myosine (MHC), l'utilisation d'anticorps monoclonaux dirigés contre diverses isoformes de MHC ainsi que le développement récent de sondes nucléiques spécifiques des MHC porcines ont permis de montrer que la diversification des fibres musculaires vers les types adultes est un phénomène complexe, matérialisé par l'expression transitoire de certaines isoformes de MHC (embryonnaire, fœtale, $\alpha$-cardiaque) et leur remplacement par les formes dites adultes (I, IIa, IIx et IIb) (Lefaucheur et al 1995, 1997 et 1998).

Les types contractiles et métaboliques des fibres musculaires évoluent fortement pendant les premières semaines de vie postnatale. Le pourcentage de fibres lentes $ß R$ augmente jusque $20 \mathrm{~kg}$ de poids vif ( 2 mois) dans tous les types de muscles, puis reste constant jusqu'aux poids commerciaux à l'abattage traditionnellement rencontrés (Lefaucheur et Vigneron 1986). En revanche, le pourcentage de fibres $\alpha \mathrm{W}$ augmente jusqu'au poids vif de $60 \mathrm{~kg}$ au détriment des fibres $\alpha \mathrm{R}$, suite à une conversion des plus grosses fibres $\alpha R$, généralement situées à la périphérie des îlots, vers le type $\alpha \mathrm{W}$ par un phénomène de dilution mitochondriale (Lefaucheur et Vigneron 1986). Le diamètre des trois types de fibres augmente jusqu'au poids commercial à l'abattage, celui des fibres $\alpha \mathrm{W}$ étant toujours supérieur à celui des fibres $\beta R$ et $\alpha R$.

Chez le porc, la mise en place et la diversification des fibres musculaires se produit essentiellement pendant les phases embryonnaire, fœtale et postnatale précoce. Ces périodes constituent donc a priori des stades privilégiés pour la manipulation des caractéristiques des fibres musculaires par les facteurs d'élevage.

\section{b / Le tissu adipeux intramusculaire}

De tous les dépôts adipeux de l'organisme, le tissu adipeux intramusculaire se met en place le plus tardivement (Lee et Kauffmann 1974). Il se développe par des phénomènes d'hypertrophie et d'hyperplasie des adipocytes intramusculaires au cours de la crois-

\section{Les fibres musculaires se différencient intensément pendant les premières semaines de vie post-natale.}


Figure 1. Evolution du nombre et du diamètre des adipocytes et de la teneur en lipides totaux du muscle Long dorsal chez le porc Large White au cours de la croissance (d'après M.J. Chora et al 1996, non publié).

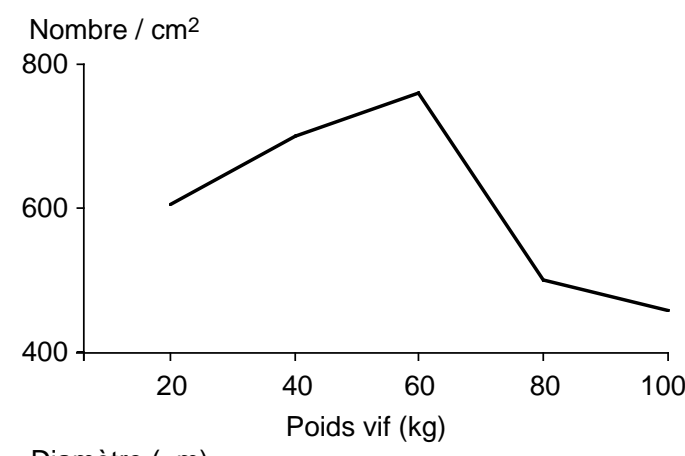

Diamètre $(\mu \mathrm{m})$

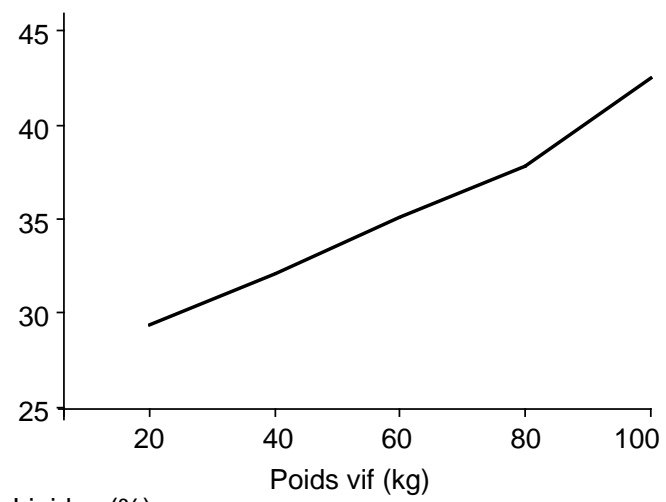

Lipides (\%)

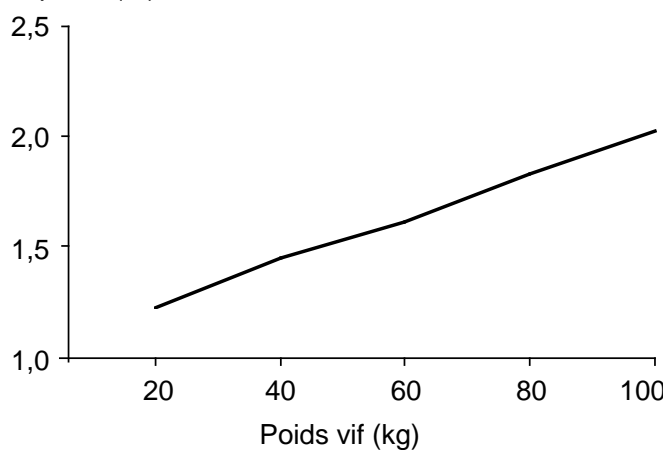

sance (Lee et Kauffmann 1974, M.J. Chora et al 1996, non publié) (figure 1). Le diamètre des adipocytes s'accroît de 20 à $100 \mathrm{~kg}$ de poids vif, alors que le nombre d'adipocytes par unité de surface du muscle augmente de 20 à $60 \mathrm{~kg}$ puis diminue jusqu'à $100 \mathrm{~kg}$ (M.J. Chora et al 1996, non publié). Cette diminution apparente s'explique par une hypertrophie importante des fibres musculaires au cours de cette période.

La teneur en lipides intramusculaires augmente au cours de la croissance (Lee et Kauffmann 1974, Malmfors et al 1978, Lazo et al 1994, Mourot et Kouba 1995, M.J. Chora et al 1996, non publié ; figure 1). L'augmentation du taux de lipides résulte essentiellement d'une augmentation du taux de triglycérides, la teneur en phospholipides restant constante au cours du développement (Lazo et al 1994). Au sein des lipides intramusculaires, le taux d'acides gras polyinsaturés diminue avec l'âge (Lazo et al 1994), en raison d'une aug- mentation de la part des acides gras d'origine endogène, saturés ou monoinsaturés, par rapport aux acides gras d'origine exogène, seule source d'acides gras polyinsaturés chez le porc.

Dans le muscle, le potentiel d'activité de l'enzyme malique, une des enzymes fournisseurs de NADPH utilisé dans la synthèse des lipides, s'accroît fortement entre 4 et 24 semaines d'âge (Lee et Kauffmann 1974, Mourot et Kouba 1995). Le coefficient de corrélation entre la teneur en lipides et le potentiel d'activité de l'enzyme malique des muscles Semi membranosus (partie blanche) et Supraspi natus (muscle rouge) est estimé à $r=0,81$ d'après Mourot et Kouba (1995) confirmant ainsi les observations de Renard et al (1992) selon lesquelles l'enzyme malique peut être considérée comme un marqueur de la capacité lipogénique du muscle. Ces résultats suggèrent qu'une meilleure connaissance de la regulation du gène de l'enzyme malique pourrait permettre de contrôler la teneur en lipides des muscles, dans le but d'améliorer les qualités organoleptiques de la viande de porc.

\section{c / Le tissu conjonctif}

La teneur et les caractéristiques du collagène intramusculaire évoluent au cours de la croissance. La teneur en collagène, très élevée à la naissance $(43 \mathrm{mg} / \mathrm{g}$ de matière sèche dans le muscle Longi ssi mus) diminue rapidement jusqu'à $30 \mathrm{~kg}$ de poids vif, puis de plus en plus lentement avec l'âge pour atteindre environ $17 \mathrm{mg} / \mathrm{g}$ de muscle sec à $105 \mathrm{~kg}$ de poids vif (165 jours) (Lebret et al 1998a). La solubilité thermique du collagène intramusculaire diminue également au cours de la croissance, mais de façon plus régulière. La proportion de collagène soluble du muscle Longi ssimus passe de 43 à $17 \%$ de la naissance à $105 \mathrm{~kg}$ de poids vif (Lebret et al 1998a). Le fait que la teneur et la solubilité thermique du collagène intramusculaire évoluent jusqu'au stade usuel d'abattage des porcs suggère que les propriétés du tissu conjonctif intramusculaire varient en fonction de l'âge des animaux à l'abattage. Une évolution similaire de la teneur et de la solubilité du collagène intramusculaire au cours de la croissance a été précédemment décrite chez le bovin (Hill 1966, Boccard et al 1979).

\section{2 / Relations entre les caractéristiques du tíssu musculaire et la qualité des viandes}

L'évolution du muscle en viande comporte deux phases : la dégradation des réserves énergétiques musculaires lors de l'établissement de la rigor mortis, puis l'évolution des protéines de structure pendant la maturation de la viande. La cinétique et l'intensité de ces réactions influencent fortement les qualités des viandes (Valin 1988). Après une présenta- 
tion des phénomènes conduisant à la transformation du muscle en viande, nous décrivons les composantes organoleptique, technologique, nutritionnelle et hygiénique des viandes de porc, en relation avec les caractéristiques du tissu musculaire.

\section{1 / E volution post-mortem du $\mathrm{pH}$}

Après l'abattage, la vitesse de chute du pH musculaire (mesurée par le pH à 45 minutes post mortem) dépend de la vitesse de diffusion des ions calcium vers le sarcoplasme, de l'activité ATPasique myofibrillaire (lent ou rapide), du pouvoir tampon du muscle et de la disponibilité en glycogène dans les fibres musculaires. Les ions calcium libérés dans le sarcoplasme vont stimuler l'activité ATPasique du complexe actomyosine, entraînant la libération de phosphate inorganique qui va stimuler la glycolyse, conduisant à la dégradation du glycogène en acide lactique. Le résultat global est une acidification progressive du muscle.

Les relations entre la vitesse de chute du $\mathrm{pH}$ et la composition en fibres musculaires sont controversées. Ainsi, aucune différence de pH 45 n'a été observée par Lefaucheur et al (1991) entre les muscles Longi ssi mus (blanc) et Semi spinalis (rouge). D'autres résultats signalent une chute du $\mathrm{pH}$ plus rapide dans le muscle Psoas major (intermédiaire) que le Longi ssimus (L. Lefaucheur, non publié) alors que Klosowska et al (1975) rapportent une baisse plus rapide du $\mathrm{pH}$ dans les muscles blancs que dans les muscles rouges. Cette controverse s'explique par le fait que, par rapport aux muscles riches en fibres rapides, les muscles riches en fibres lentes ont une activité ATPasique plus faible, ce qui ralentit la vitesse de chute $\mathrm{du} \mathrm{pH}$, mais un pouvoir tampon inférieur, ce qui augmente la vitesse de chute du pH (Talmant et al 1986).

L'amplitude de chute du $\mathrm{pH}$, mesurée par le $\mathrm{pH} 24$ heures ( $\mathrm{pH}$ ultime ou $\mathrm{pHu}$ ) est fonction de la quantité de glycogène transformé en acide lactique et du pouvoir tampon du muscle. Cette amplitude est essentiellement dépendante du taux de glycogène musculaire au moment de l'abattage. Les fibres blanches étant plus riches en glycogène que les fibres rouges, le pH ultime est d'autant plus bas que la proportion de fibres glycolytiques est élevée (Laborde et al 1985, Monin et al 1987).

\section{2 / Maturation de la viande}

La phase de maturation va conduire à une augmentation progressive de la tendreté de la viande, en raison de modifications qui affectent principalement le compartiment myofibrillaire. En effet, le collagène ne subit pas de modifications importantes pendant la phase de maturation; alors que la structure myofibrillaire est profondément remaniée sous l'action d'enzymes protéolytiques endogènes (Valin 1988, Ouali 1991). A ce jour, deux systèmes protéolytiques identifiés dans le tissu musculaire semblent principalement impliqués dans les processus de maturation post mortem : le système calpaïnes/calpastatine, et le système cathepsines/cystatines. Les calpaïnes sont des protéases neutres calciumdépendantes, et les cathepsines des protéases acides Iysosomiales (Valin 1988, Ouali 1991). S'agissant d'un phénomène enzymatique, la vitesse de maturation est fonction de la température, mais également du $\mathrm{pH}$ du muscle. Plusieurs études rapportent que la vitesse de maturation augmente avec l'activité ATPasique myofibrillaire et le caractère glycolytique des muscles (Ouali 1991), vraisemblablement en raison du rapport calpaïne/calpastatine plus élevé dans les muscles glycolytiques rapides que dans les muscles oxydatifs lents (Ouali et Talmant 1990). Accroître la proportion de fibres $\alpha \mathrm{W}$ peut donc présenter des avantages chez les espèces à vitesse de maturation lente, telle que l'espèce bovine (Dransfield et al 1981). Cependant, une étude récente rapportée par Zamora et al (1996) chez le bovin montre que l'augmentation de la vitesse de chute post mortem du pH entraîne une diminution de la tendreté instrumentale du muscle Longissimus après 14 jours de maturation à $4^{\circ} \mathrm{C}$. Chez le porc, une espèce à vitesse de maturation post mortem rapide, une vitesse de chute trop rapide $\mathrm{du} \mathrm{pH}$ est jugée responsable du manque de maturation des viandes PSE (Pale, Soft and Exudative, pour pâle, molle et exudative), et des défauts de tendreté qui en découlent (Minelli et al 1995). En conséquence, accroître la proportion de fibres $\alpha \mathrm{W}$, comme c'est le cas chez les porcs PSE, est également susceptible de diminuer l'amplitude de la maturation post-mortem de la viande et la tendreté. En conséquence, l'identification du type de fibre musculaire à rechercher pour améliorer la tendreté de la viande nécessite donc des travaux complémentaires.

Les industriels de la salaison du jambon déclarent être confrontés aujourd'hui à un autre défaut de qualité de viande, dénommé le jambon "pommade". II s'agirait d'une altération de la structure musculaire touchant essentiellement le muscle Semi membranosus dans sa partie profonde (près de l'os) mais également les autres muscles dans les cas les plus graves. Ce défaut est visible lors du désossage des jambons avant transformation, les parties musculaires atteintes apparaissant décolorées, molles et sans structure (viande déstructurée). Sur les jambons cuits, les parties dégénérées $s^{\prime}$ effritent et forment des trous lors du tranchage, entraînant des pertes économiques considérables. Ni la fréquence, ni l'origine de ce défaut n'ont été établies à ce jour. L'identification des causes d'apparition et surtout la mise en œuvre de solutions permettant de pallier ce problème constituent l'une des priorités de la filière porcine aujourd'hui en matière de qualité de viande.

\section{3 / Qualité organoleptique}

\section{a / Aspect visuel}

Le consommateur recherche en général une viande ni trop pâle, ni trop foncée, et de couleur homogène. La couleur dépend de la quan- 
Tableau 2. Corrélations simples entre la teneur en lipides intramusculaires et certaines composantes de la qualité organoleptique de la viande de porc.

\begin{tabular}{|l|c|c|c|c|c|}
\hline Référence & $\begin{array}{c}\text { Force } \\
\text { de cisaillement }\end{array}$ & Tendreté & Jutosité & Flaveur & $\begin{array}{c}\text { Acceptabilité } \\
\text { globale }\end{array}$ \\
\hline Cromwell et al (1978) & $-0,28$ & 0,17 & 0,50 & 0,10 & 0,31 \\
Wood et al (1986) & --- & 0,13 & 0,31 & 0,17 & 0,22 \\
De Vol et al (1988) & $-0,29 * *$ & $0,32^{* * *}$ & $0,21^{*}$ & $0,23 *$ & --- \\
Ramsey et al (1990) & $-0,24^{*}$ & --- & -- & -- & --- \\
Hodgson et al (1991) & $-0,48^{* *}$ & --- & $0,65^{* *}$ & --- & $0,51^{* *}$ \\
Eikelenboom et al (1996a) & 0,01 & 0,30 & $0,33^{*}$ & 0,30 & --- \\
Wood et al (1996) & -- & 0,09 & 0,36 & 0,22 & 0,24 \\
\hline
\end{tabular}

$* \mathrm{P}<0,05 ; * * \mathrm{P}<0,01 ; * * * \mathrm{P}<0,001$

tité de myoglobine, liée au pourcentage de fibres rouges, de l'état chimique de ce pigment, ainsi que de la structure du muscle réfléchissant la lumière. L'abaissement du pH augmente la quantité d'eau extracellulaire et, en conséquence, la réflexion de la lumière incidente (Offer et al 1988), ce qui confère un aspect clair aux viandes à bas $\mathrm{pH}$.
Les tissus adipeux intermusculaire (marbré) et intramusculaire (persillé) influencent également l'acceptabilité de la viande par le consommateur qui n'apprécie pas une quantité trop importante de gras visible, même si la teneur en lipides intramusculaires améliore les autres composantes de la qualité organoleptique (tableau 2). Une étude réalisée

Figure 2. Influence de la teneur en lipides du muscle Long dorsal sur l'acceptabilité de la viande par les consommateurs (d'après Fernandez et al 1996).
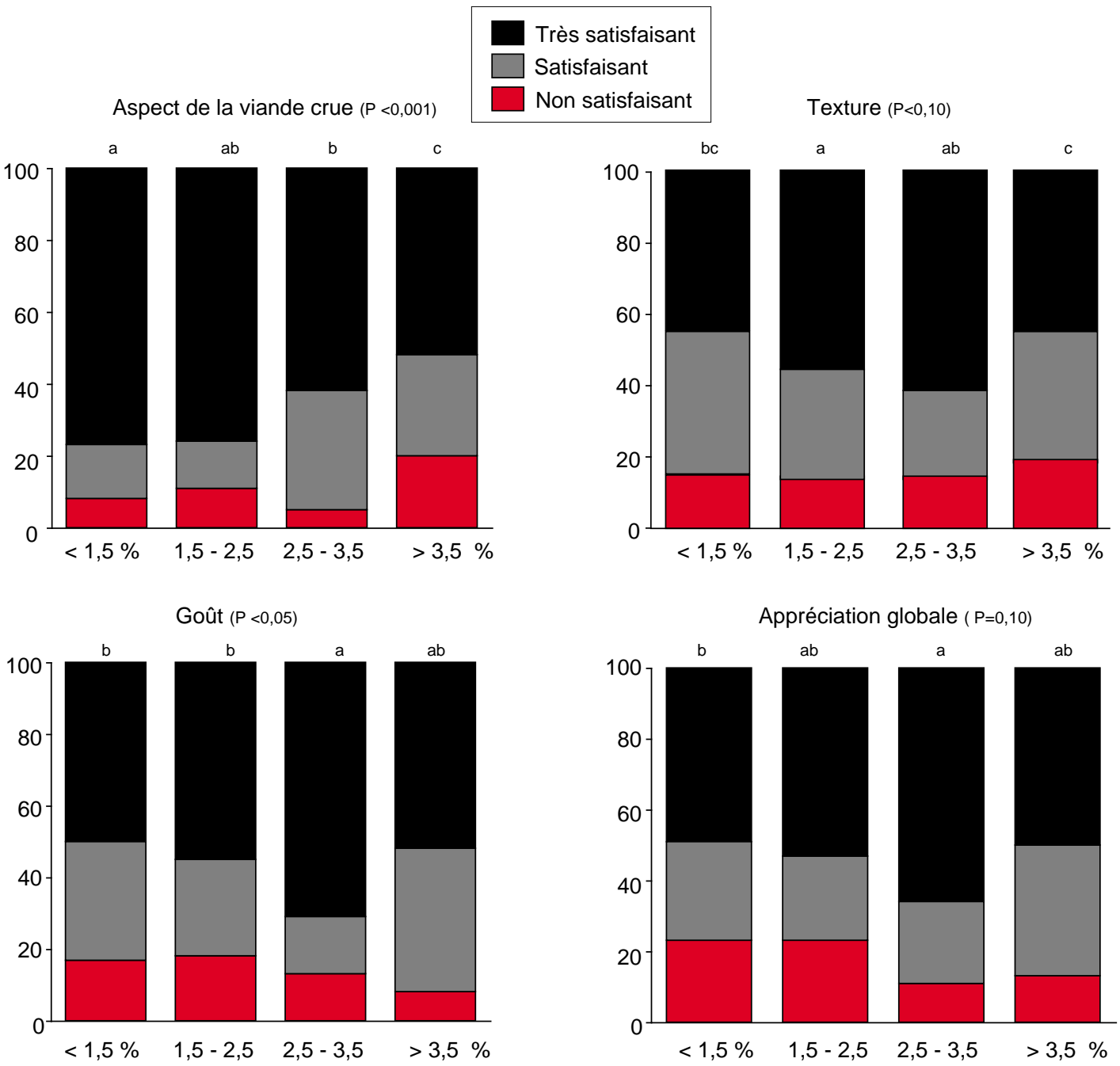

Teneur en lipides du muscle Long dorsal 
par Fernandez et al (1996) confirme ce paradoxe : les consommateurs ne souhaitent pas voir de gras dans la viande avant consommation, alors que l'appréciation de la viande après consommation augmente avec le taux de lipides intramusculaires jusqu'à une teneur en lipides comprise entre 2,5 et 3,5\% (figure 2).

\section{b / Tendreté}

La tendreté de la viande, qui correspond à la facilité avec laquelle celle-ci se laisse trancher ou mastiquer, dépend de la quantité, de la répartition et du degré de polymérisation du collagène musculaire, ainsi que de la structure myofibrillaire (Monin 1983). Concernant la composante collagénique, il semble que les proprietés du collagene (solubilité thermique, nature des liaisons intermoléculaires) soient plus importantes que le taux de collagène dans la détermination de la tendreté (Bailey et Light 1989). Chez le porc, on considère généralement que la composante collagénique est peu impliquée dans la détermination de la tendreté de la viande fraîche, en raison de l'âge relativement jeune des animaux à l'abattage ( 5 à 6 mois au poids commercial de $105 \mathrm{~kg}$ ). En revanche, cette composante influence les propriétés des produits transformés cuits, la gélatine obtenue suite à la solubilisation du collagène musculaire lors des traitements thermiques intervenant dans la cohésion et la texture des produits.

La composante myofibrillaire joue un rôle prépondérant dans la détermination de la tendreté de la viande fraîche chez le porc. Les propriétés des myofibrilles dépendent essentiellement de l'évolution post mortem du $\mathrm{pH}$ et des réactions protéolytiques intervenant pendant la maturation (Monin 1983, Valin 1988). Les travaux de Minelli et al (1995) et Monin et al (1999) montrent que la vitesse de chute du $\mathrm{pH}$ constitue le principal déterminant de la résistance myofibrillaire et de la dureté de la viande de porc, le pH ultime ayant un effet moins important. La tendreté augmenterait avec la durée de conditionnement de la viande (Wood et al 1996).

Une liaison positive a été rapportée entre l'intensité du métabolisme oxydatif et la tendreté du muscle Longissimus (EssenGustavsson et Fjelkner-Modig 1985). Par ailleurs, plusieurs travaux ont montré une amélioration de la tendreté de la viande de porc avec l'augmentation de la teneur en lipides intramusculaires (Cromwell et al 1978, De Vol et al 1988, Touraille et al 1989, Fernandez et al 1996), même si cette relation n'est pas toujours très marquée (tableau 2).

Plusieurs études signalent une liaison négative entre le diamètre des fibres et la tendreté (Carpenter et al 1963 chez le porc, Joubert 1956 chez le mouton). Ainsi, la recherche d'un nombre total de fibres élevé associé à un diamètre des fibres relativement faible constitue a priori une voie permettant de concilier les impératifs quantitatifs et qualitatifs de la production de viande de porc.

\section{c / J utosité}

La jutosité, ou impression de libération de jus au cours de la mastication, est liée à la quantité d'eau libre subsistante dans la viande et à la sécrétion de salive stimulée essentiellement par les lipides (Girard et al 1988). Elle varie avec le pouvoir de rétention d'eau (PRE) de la viande, les pertes à la cuisson et la présence de lipides. L'évolution post mortem du $\mathrm{pH}$, qui influence fortement le PRE et les pertes à la cuisson (Monin 1983 et 1988) joue un rôle important dans la détermination de la jutosité de la viande. Eikelenboom et al (1996a) évaluent à 0,68 le coefficient de corrélation entre le pHu et la jutosité de la viande. De nombreux travaux montrent que les lipides intramusculaires influencent favorablement la jutosité de la viande (Cromwell et al 1978, Wood et al 1986, De Vol et al 1988, Touraille et al 1989, Hodgson et al 1991, Eikelenboom et al 1996b), le coefficient de corrélation entre le taux de lipides intramusculaires et la jutosité variant selon les études entre 0,21 et 0,65 (tableau 2).

\section{d / F laveur}

La flaveur correspond aux perceptions olfactives et gustatives lors de la dégustation. Elle dépend essentiellement de la composante lipidique (tableau 2; De Vol et al 1988, Touraille et al 1989, Gandemer et al 1990). La teneur optimale en lipides intramusculaires pour l'obtention de caractéristiques sensorielles favorables est de 2,5 à 3,0\% dans le muscle Longissimus de porc (De Vol et al 1988, Fernandez et al 1996). Parmi la composante lipidique, un rôle important est attribué aux phospholipides dans le développement de la flaveur caractéristique de la viande cuite (Mottram et $\mathrm{Edwards}$ 1983). En conséquence, la typologie d'un muscle peut influencer la flaveur de la viande en raison des différences de teneur et de composition en phospholipides selon le type métabolique des muscles (Leseigneur-Meynier et Gandemer 1991). Chez le mouton, Valin et al (1982) ont montré que l'intensité de la flaveur est directement liée au caractère rouge de la viande, en particulier au pourcentage de fibres lentes. Le même phénomène pourrait exister chez le porc, mais n'a pas fait l'objet d'études.

D'autre part, la nature des lipides intramusculaires agit sur l'aptitude à la conservation de la viande (Monahan et al 1990, Mourot et al 1992a), en raison de la sensibilité des acides gras polyinsaturés (AGPI) au phénomène de peroxydation (rancissement). Les produits de la filière porcine sont particulièrement sensibles au rancissement, en raison de leurs teneurs relativement élevées en AGPI et des faibles teneurs des viandes en agents antioxydants (Mourot et al 1992a, Dirinck et De Winne 1995).

Chez le porc, un défaut de flaveur particulier est dû à l'utilisation des viandes de porcs mâles entiers, qui peuvent présenter des défauts d'odeur lors de la cuisson ou, dans les cas les plus défavorables, lors de la consommation. Deux composés majeurs mis en évi- 
dence dans les graisses, l'androsténone et le scatol, sont actuellement tenus pour responsables de ces défauts d'odeur sexuelle (Bonneau 1988).

En conclusion, l'influence de la teneur, et dans une moindre mesure de la nature des lipides intramusculaires sur les différentes composantes de la qualité organoleptique des viandes est relativement bien établie. Par contre, les relations entre les caractéristiques des fibres musculaires : type, diamètre, nombre total de fibres et les qualités sensorielles ne sont pas encore bien identifiées. Un programme de recherches en cours à l'INRA a pour objectif d'évaluer l'influence des caractéristiques contractiles et métaboliques des fibres musculaires chez le porc sur les qualités des viandes, en réalisant une sélection divergente sur la surface relative occupée par les fibres de type $\beta R$ dans le muscle Longi ssi mus au sein d'une population Large White. Ces travaux permettront d'apporter des réponses sur les relations entre les caractéristiques des fibres musculaires et les différentes composantes de la qualité.

\section{4 / Qualité technologique}

La qualité technologique de la viande correspond à ses aptitudes à subir une transformation. Dans le cas de la filière porcine dans laquelle de multiples procédés de transformation sont mis en œuvre, la qualité de la matière première doit être définie par rapport à l'utilisation envisagée. Nous prendrons ici l'exemple de la transformation en jambon cuit, qui constitue un des produits de la filière les plus importants du point de vue économique.

Le pouvoir de rétention en eau (PRE) de la viande fraîche est une caractéristique essentielle pour la fabrication de jambon cuit. Le coefficient de corrélation entre le PRE et le rendement de fabrication du jambon cuit est estimé à 0,70 selon Goutefongea et al (1978). Le PRE, qui correspond à la capacité des $20 \%$ de protéines musculaires à retenir les $75 \%$ d'eau présents, est fortement influencé par la vitesse et l'amplitude de chute du $\mathrm{pH}$ post mortem (Monin 1983). Une chute trop rapide du $\mathrm{pH}$ combinée à une température élevée provoque la dénaturation des protéines, conduisant à une réduction du PRE et à la production d'une viande de type PSE (Goutefongea et al 1978). L'abaissement du $\mathrm{pH}$ ultime diminue la charge nette des protéines, entraînant une baisse du PRE qui est minimal lorsque le pH est égal au pH isoélectrique $(\mathrm{pHi})$ des protéines, soit environ 5,3 (Goutefongea et al 1978, Monin 1988). Pour des $\mathrm{pH}$ ultimes qui s'éloignent $\mathrm{du} \mathrm{pHi}$, on observe une liaison positive entre le $\mathrm{pH}$ ultime et le rendement de fabrication du jambon cuit. La présence de glycogène résiduel dans le muscle 24 heures après l'abattage entraîne également une diminution du rendement de fabrication du jambon cuit, en raison de la forte capacité du glycogène à fixer de l'eau qui sera libérée lors de la cuisson parallèlement à l'hydrolyse du glycogène (Monin 1988). Le PRE serait relié négativement au pourcentage de fibres blanches et/ou au diamètre de ces fibres (Klosowska et al 1975).

\section{5 / Qualité nutritionnelle}

En matière de santé publique, les nutritionnistes recommandent une diminution de la part des lipides dans l'alimentation et une réduction des apports en acides gras saturés au profit des acides gras mono et polyinsaturés, en particulier les acides gras polyinsaturés n-3 (comme l'acide linolénique C18:3), en raison de leur effet protecteur vis-à-vis des maladies cardiovasculaires. De plus, l'acide linolénique et l'acide linoléique (C18:2, n-2) sont des acides gras indispensables à l'Homme car l'organisme ne peut les synthétiser.

La viande de porc est souvent jugée grasse par le corps médical ou les consommateurs, en raison d'une confusion faite entre l'adiposité globale de la carcasse et la teneur en lipides de la fraction maigre de la viande. En réalité, les plus gros muscles du porc sont pauvres en lipides $(1,5$ à $2 \%$ dans le Longi ssi mus, contre 5 à $6 \%$ dans le muscle équivalent chez le bovin). Chez le porc, les lipides intramusculaires présentent un rapport acides gras polyinsaturés / saturés plus élevé que celui observé chez les ruminants, en raison notamment de teneurs relativement élevées en acides gras essentiels C18:2 et C18:3, conférant à la viande des qualités nutritionnelles intéressantes (Wood et Enser 1996). Sur cet aspect, il est important de rappeler que les animaux monogastriques présentent la particularité de déposer les acides gras alimentaires dans leurs tissus sans modification chimique, contrairement aux ruminants qui ont la capacité de saturer les acides gras d'origine alimentaire. Ainsi chez le porc, il est possible de modifier, dans une certaine mesure, la composition en acides gras de la viande par l'alimentation, dans le but d'améliorer la qualité nutritionnelle des produits.

\section{6 / Qualité hygiénique}

La qualité hygiénique de la viande constitue l'exigence élémentaire du consommateur. Elle peut être altérée par la prolifération de microorganismes néfastes et/ou la présence de composés toxiques. La viande peut être contaminée par des microorganismes à différentes étapes de la chaîne de transformation. Le contrôle des proliférations microbiennes dépend avant tout du respect de la chaîne du froid, cependant plusieurs facteurs peuvent également influencer la multiplication microbienne. Ainsi, un pH ultime élevé favorise le développement de bactéries putréfiantes et freine la capacité de pénétration du sel dans la viande (Goutefongea et al 1978). En conséquence, les muscles riches en fibres rouges lentes, pauvres en glycogène, sont plus exposés aux proliférations bactériennes néfastes.

L'utilisation de certaines molécules en élevage (antibiotiques, facteurs de croissance), qui peut entraîner la présence de résidus dans les viandes, devient une préoccupation grandissante pour le public (Tarrant 1998). L'évolution possible de la demande des consommateurs vers une viande exempte de résidus pourrait avoir des conséquences considérables sur l'ensemble des acteurs de la filière. 


\section{3 / F acteurs de variation de la qualité du tissu musculaire}

Ce chapitre traite de l'effet de différents facteurs d'élevage non génétiques sur les caractéristiques du tissu maigre et la qualité de la viande. Deux composantes musculaires retiendront principalement notre attention, les lipides et les fibres musculaires.

\section{1 / La localisation anatomique}

Les différents muscles de la carcasse présentent des caractéristiques chimiques, biochimiques et des compositions en fibres très différentes. Les muscles du porc contiennent généralement les trois types de fibres ( $\beta R, \alpha R$ et $\alpha W)$, mais dans des proportions variables. Le Longissimus et la plupart des gros muscles du jambon sont des muscles blancs rapides : Longi ssi mus : $10 \% \beta R, 15 \% \alpha \mathrm{R}$, $75 \% \alpha W$ (Lefaucheur et al 1992), Biceps femoris : $22 \%$ ßR, $8 \% \alpha \mathrm{R}, 70 \% \alpha \mathrm{W}$ (Petersen et al 1994), alors que les muscles de la partie antérieure du corps et les muscles profonds situés près des os sont généralement rouges et riches en fibres lentes (partie rouge du Semi tendinosus, et Semi spi nalis : $40 \%$ ßR, $60 \% \alpha R$ (Lefaucheur et al 1992)). Les teneurs en lipides varient également considérablement selon les muscles, mais semblent indépendantes de leur type métabolique et contractile. Les variations des caractéristiques musculaires en fonction de la localisation anatomique influencent les qualités organoleptiques et technologiques des viandes et, en conséquence, l'utilisation et la valeur économique des morceaux de découpe.

\section{2 / Caractéristiques de l'animal à l'abattage}

\section{a / L'âge et le poids}

Les teneurs en lipides et en matière sèche du muscle augmentent avec l'âge et le poids des animaux (Malmfors et al 1978, Desmoulin et al 1983, Lazo et al 1994, Cisneros et al 1996a, Candek-Potokar et al 1998). Pour un même poids à l'abattage, une augmentation de l'âge obtenue par le biais d'une restriction alimentaire globale entraîne une diminution du taux de lipides intramusculaires (CandekPotokar et al 1998 ; figure 3) et une augmentation du diamètre des fibres du muscle Longi ssi mus en relation avec l'augmentation du taux de muscle de l'animal (Lefaucheur 1983). Aucun effet sur le type contractile et métabolique des fibres n'a eté observé.

La qualité technologique de la viande n'est pas modifiée par une augmentation du poids des porcs à l'abattage de 100 à $120-130 \mathrm{~kg}$ (Desmoulin et al 1983, Monin 1983, CandekPotokar et al 1998). Plusieurs études montrent qu'une telle augmentation du poids à l'abattage ne modifie pas non plus la qualité organoleptique de la viande, évaluée par des mesures physiques ou sensorielles (Malmfors et al 1978, Desmoulin et al 1983, Candek-
Figure 3. Variation de la teneur en lipides du muscle Long dorsal en fonction du poids vif et de l'âge des animaux à l'abattage (d'après Candek-Potokar et al 1998).

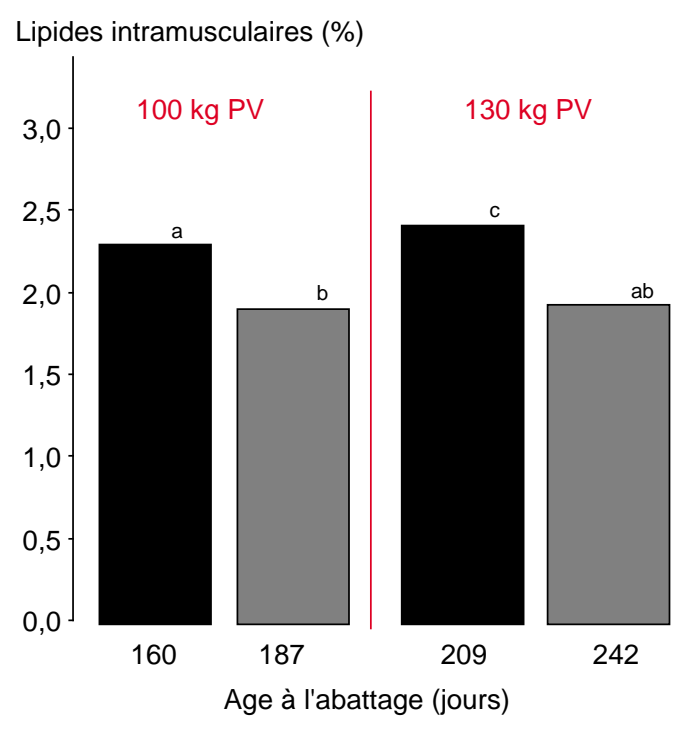

Alimentation à volonté

Rationnement (- $30 \%)$

Potokar et al1998), alors que Ellis et al (1996) rapportent une augmentation de la force de cisaillement et une diminution de la tendreté, de la jutosité et de l'acceptabilité globale de la viande avec l'augmentation du poids à l'abattage de 80 à $120 \mathrm{~kg}$. Chez les types génétiques à forte vitesse de croissance, l'absence d'effet du poids vif sur la flaveur et la jutosité pourrait résulter d'une trop faible variation de la teneur en lipides intramusculaires entre les stades d'abattage considérés, en raison du développement tardif du tissu adipeux intramusculaire. Une augmentation plus importante du poids à l'abattage de 100 à $160 \mathrm{~kg} \mathrm{~s}$ 'accompagne d'une légère diminution du $\mathrm{pH}$ ultime et de la tendreté du muscle Longi ssi mus, malgré l'augmentation de sa teneur en lipides (Cisneros et al 1996a).

\section{b / L'adiposité de la carcasse}

Pour un génotype donné, la teneur en lipides intramusculaires varie généralement dans le même sens que l'adiposité globale de la carcasse (Desmoulin et al 1983, Cisneros et al 1996a). En conséquence, tout facteur d'élevage modifiant l'adiposité globale de la carcasse peut influencer la teneur en lipides des muscles. Un des objectifs des recherches entreprises actuellement sur la mise en place et le développement des tissus adipeux externe et intramusculaire chez le porc est de parvenir à augmenter le taux de lipides intramusculaires sans modifier l'adiposité globale de la carcasse.

\section{c / Le type sexuel}

Les teneurs en lipides intramusculaires des femelles et des mâles entiers sont équivalentes et inférieures à celles des mâles castrés
A même poids à l'abattage, un âge plus élevé correspond à une moindre teneur en lipides. 
(Malmfors et al 1978, Barton-Gade 1987, Girard et al 1988, Larzul et al 1997), en liaison avec la plus forte adiposité globale de ces derniers. Leur teneur en lipides intramusculaires supérieure s'accompagne d'un degré d'insaturation des lipides plus faible, permettant une meilleure aptitude des viandes à la conservation (Malmfors et al 1978, Girard et al 1988).

Des études montrent une absence d'effet du sexe sur le nombre total de fibres musculaires chez le porc (Staun 1963, Larzul et al 1997). En conséquence, la croissance musculaire plus élevée des mâles entiers et des femelles que des castrats devrait résulter d'une plus grande aire de section transversale (AST) des fibres chez les mâles entiers et les femelles, or la bibliographie est controversée à ce sujet : I'AST est supérieure chez les femelles par rapport aux mâles castrés selon Miller et al (1975) et Larzul et al (1997), alors que Staun (1963) n'observe pas de différence. Le type sexuel (femelle vs mâle castré) n'influence ni la composition en types de fibres musculaires (Larzul et al 1997), ni les activités enzymatiques métaboliques du muscle (Monin et al 1986, Larzul et al 1997). En revanche, les mâles entiers présentent une surface relative occupée par les fibres lentes dans le muscle Longi ssi mus supérieure à celle rencontrée chez les femelles et les mâles castrés (Lefaucheur, non publié).

Concernant la qualité technologique de la viande, l'effet du sexe sur le $\mathrm{pH}$ ultime est controversé. Plusieurs études rapportent un $\mathrm{pH}$ ultime identique chez les mâles castrés et les femelles (Desmoulin et al 1983, BartonGade 1987, Cisneros et al 1996a), alors que Larzul et al (1997) observent un pHu plus bas chez les femelles, en accord avec un potentiel glycolytique plus élevé. Selon Fernandez et Tornberg (1991), les mâles entiers présentent un $\mathrm{pHu}$ plus élevé que les castrats et les femelles, en raison d'une plus grande mobilisation du glycogène musculaire avant l'abattage consécutive à une excitation physique plus importante. Les autres critères de qualité technologique de la viande ne sont pas différents entre les femelles et les mâles castrés (Desmoulin et al 1983, Castell et al 1994, Cisneros et al 1996a).

La réflectance de la viande issue de mâles castrés serait plus élevée que celle des femelles selon Renou et al (1985), alors que Cisneros et al (1996a) et Larzul et al (1997) n'observent pas cet effet. Les mesures physiques ou sensorielles de qualité organoleptique de la viande sont généralement équivalentes entre les mâles castrés et les femelles (Malmfors et al 1978, Desmoulin et al 1983, Cisneros et al 1996a, Ellis et al 1996), toutefois la viande de mâles castrés est parfois jugée plus tendre et de flaveur plus intense (Bereskin et al 1978, Castell et al 1994). En revanche, les viandes de porcs mâles entiers sont quelquefois jugées plus dures (Desmoulin et al 1983, Barton-Gade 1987). Cependant, le principal problème lié à l'emploi des viandes de porcs mâles entiers reste celui des défauts d'odeur sexuelle.

\subsection{Caractéristiques de I'animal à la naissance}

Le poids de l'animal à la naissance peut modifier ses caractéristiques au stade de l'abattage. En effet, selon Powell et Aberle (1980), il existerait un poids à la naissance critique (environ $1000 \mathrm{~g}$ chez des porcelets croisés Landrace $x$ Yorkshire) au-dessous duquel le potentiel de croissance musculaire est définitivement réduit (animaux dénommés "culots de portée"). Ceci est la conséquence d'une réduction du nombre total de fibres musculaires (Hegarty et Allen 1978, Powell et Aberle 1980), qui touche principalement la seconde génération de fibres (Wigmore et Stickland 1983). Le format adulte et la vitesse de croissance de ces animaux sont inférieurs à ceux des animaux normaux (Hegarty et Allen 1978, Powell et Aberle 1980). Au moment de l'abattage, à même poids vif, les animaux de très faible poids à la naissance (inférieur à $850 \mathrm{~g}$ ) présentent des carcasses plus grasses, une aire de section transversale du muscle Longi ssimus inférieure et un taux de lipides intramusculaires supérieur par rapport aux animaux normaux (Powell et Aberle 1980), ainsi qu'un diamètre plus élevé des fibres musculaires dans les muscles Semitendinosus et Psoas major (Hegarty et Allen 1978). Les variations des caractéristiques musculaires observées chez les animaux de très faible poids à la naissance pourraient se répercuter sur les propriétés des viandes, toutefois, à notre connaissance, ceci n'a pas fait l'objet d'études.

\section{4 / L'alimentation}

\section{a / Niveau alimentaire}

Une diminution globale du niveau alimentaire au cours de la croissance diminue l'adiposité de la carcasse au profit des dépôts musculaires, lorsqu'on compare les animaux au même poids. En conséquence, la teneur en lipides intramusculaires est réduite chez les animaux restreints pour un poids à l'abattage donné (Wood et al 1996, Candek-Potokar et al 1998 ; figure 3). Appliquée chez le jeune, la restriction alimentaire provoque une diminution de la teneur en lipides intramusculaires liée à une réduction de la taille et du nombre des adipocytes, que l'on compare les animaux à âge constant ( 24 semaines) ou à poids constant ( $80 \mathrm{~kg}$ de poids vif) (Lee et al 1973a et 1973b). L'effet de la restriction alimentaire sur la cellularité du tissu adipeux intramusculaire du porc en croissance-finition n'a pas été étudié. Consécutivement à la diminution de la teneur en lipides totaux du muscle, les porcs restreints présentent une proportion d'acides gras polyinsaturés dans les lipides totaux supérieure à celle observée chez les porcs nourris à volonté (Cameron et Enser 1991).

La composition en fibres du muscle Longi ssi mus n'est pas modifiée par une restriction alimentaire globale de $8 \%$ (Lebret et al 1998b) ou de $30 \%$ (Lefaucheur 1983) pendant l'engraissement. Cependant, la restriction entraîne un accroissement du diamètre 
des fibres musculaires dans le Longissimus, conduisant à une augmentation de la surface de noix du muscle (Lefaucheur 1983). Chez les animaux restreints, une augmentation de la surface relative des fibres rouges rapides au détriment des fibres blanches a été observée dans le muscle Longi ssi mus (Lebret et al 1998b).

La qualité technologique de la viande semble peu affectée par une restriction alimentaire globale (Cromwell et al 1978, Candek-Potokar et al 1998). Concernant la qualité sensorielle, Ellis et al (1996) rapportent un effet négatif de la restriction alimentaire globale (20\% par rapport au niveau à volonté) sur la tendreté, la jutosité et l'acceptabilité de la viande d'animaux abattus au même poids vif, alors que d'autres travaux ne montrent pas d'effet significatif (Wood et al 1996, Candek-Potokar et al 1998).

\section{b / E quilibre entre les principa ux nutriments (protéines, glucides, lipides)}

Les efforts de la sélection en faveur d'une production accrue de viande maigre ont entraîné un accroissement des besoins en protéines des porcs relativement aux besoins en énergie (Henry 1993). Ainsi, un niveau protéique élevé en engraissement permet aux animaux d'exprimer leur potentiel de croissance musculaire.

La distribution d'un régime déficitaire en protéines et en lysine, mais non limitant en énergie, pendant la période de croissance finition s'accompagne d'une augmentation de l'adiposité de l'animal et de la teneur en lipides intramusculaires (Bereskin et al 1978 Karlsson et al 1992, Castell et al 1994). Concernant la composition en fibres musculaires, Karlsson et al (1992) n'observent pas d'effet d'une réduction du taux protéique de la ration sur les pourcentages et la surface relative des différents types de fibres dans le muscle Longi ssimus. Le pH ultime et le pouvoir de rétention d'eau n'étaient pas affectés non plus. Certaines études mettent en évidence une amélioration de la qualité sensorielle chez les animaux ayant recu un régime déficitaire en protéines : diminution de la force de cisaillement (Karlsson et al 1992), augmentation de la tendreté et de la jutosité (Bereskin et al 1978, Castell et al 1994). Malgré des variations de teneur en lipides intramusculaires, ces différents travaux ne rapportent pas de modification de la flaveur.

Si la distribution d'un régime déficitaire en protéines pendant l'engraissement augmente la teneur en lipides intramusculaires et semble bénéfique du point de vue de la qualité sensorielle de la viande, cette pratique n'est pas appliquée en raison de l'augmentation de l'adiposité globale de la carcasse. Afin de limiter cet inconvénient, Cisneros et al (1996b) ont distribué un régime déficitaire en protéines pendant une courte période (quelques semaines) en fin d'engraissement. Ils observent une augmentation de 3,8 à 5,7\% de la teneur en lipides du muscle Longi ssimus, ainsi qu'un accroissement modéré de l'épaisseur de la bardière (Cisneros et al 1996b). Toutefois, ces résultats ont été obtenus sur des génotypes de porcs américains présentant des taux de lipides intramusculaires élevés et nécessiteraient d'être validés sur des génotypes présentant des teneurs inférieures en lipides intramusculaires.

\section{c / Composition en acides gras}

Pour des régimes alimentaires isoénergétiques, isoprotéiques et isolipidiques, la nature des acides gras de la ration peut modifier les caractéristiques de la carcasse et de la fraction maigre de la viande. L'incorporation d'une matiere grasse insaturée (huile de colza) par rapport à une matière grasse saturée (lait de vache), comme l'augmentation de la teneur en acide linoléique dans les lipides alimentaires ne modifient pas la vitesse de croissance, mais diminuent le taux de muscle et augmentent l'adiposité des animaux (Mourot et al 1991 et 1995). Le régime riche en acides gras polyinsaturés n'influence pas significativement la teneur en lipides du muscle Longissimus, mais conduit à un potentiel d'activité lipogénique supérieur de ce muscle (Mourot et al 1995).

La composition en acides gras des lipides intramusculaires est influencée par la composition en acides gras du régime (Girard et al 1988, Mourot et al 1991, 1995), toutefois cette relation est moins forte que dans les tissus adipeux externes (Girard et al 1988, Mourot et al 1991). Cette particularité de l'animal monogastrique permet de modifier par voie nutritionnelle la composition en acides gras de la viande afin de répondre à la demande des consommateurs et des nutritionnistes qui recherchent un taux d'acides gras polyinsaturés élevé. Cependant, le risque de développement des réactions d'oxydation des acides gras polyinsaturés, qui peuvent conduire au rancissement, est alors augmenté. Selon Van Oeckel et al (1996), la teneur en acide linolénique de la viande peut être augmentée jusqu'à $3 \%$ des acides gras totaux sans conséquences sur la qualité organoleptique, et Ahn et al (1996) rapportent qu'une teneur en acide linolénique de $12 \%$ entraîne le développement de réactions d'oxydation et produit un effet négatif sur l'acceptabilité de la viande cuite lorsqu'elle n'est pas conservée sous vide.

\section{d / Apports vitaminiques}

Les lipides de la viande de porc étant relativement sensibles à l'oxydation, l'incorporation de vitamine $\mathrm{E}$, un antioxydant, a été envisagée dans l'alimentation. Cette vitamine, presente dans les muscles au niveau des membranes cellulaires, permet de limiter l'oxydation des acides gras polyinsaturés au cours de la conservation de la viande (Monahan et al 1990, Mourot et al 1992a). Des teneurs élevées en vitamine $\mathrm{E}$ dans l'alimentation $(200 \mathrm{mg} / \mathrm{kg}$ ) pendant la phase de croissance - finition ( 35 à $100 \mathrm{~kg}$ de poids vif) ralentissent la perte de couleur rouge de la viande au cours de la conservation, en raison

\section{La qualité technologique de la viande semble peu affectée par une restriction alimentaire globale.}


de l'effet protecteur de la vitamine E vis-à-vis de l'oxydation de la myoglobine (Monahan et al 1992). Cheah et al (1995) rapportent une réduction de plus de $50 \%$ de la perte en eau de la viande fraîche par exsudation chez les animaux supplémentés en vitamine $E$ entre 50 et $95 \mathrm{~kg}$ de poids vif, consécutivement à l'augmentation de la stabilité des membranes cellulaires.

L'apport alimentaire de vitamine $E$ influence également les qualités organoleptiques des viandes. Dirinck et De Winne (1995) rapportent un effet positif d'une supplémentation à $200 \mathrm{mg} / \mathrm{kg}$, comparé à $60 \mathrm{mg} / \mathrm{kg}$ entre 45 et $100 \mathrm{~kg}$ de poids vif, pour le goût frais, la tendreté et la jutosité de la viande après conservation pendant 5 jours à $4^{\circ} \mathrm{C}$.

L'influence d'une supplémentation du régime alimentaire en acide ascorbique, ou vitamine $C$, sur la qualité de la viande a été évaluée. Un apport de $250 \mathrm{mg} / \mathrm{kg}$ d'aliment entre 35 et $100 \mathrm{~kg}$ de poids vif diminue la réflectance de la viande qui apparaît alors plus sombre et entraîne une augmentation du pH ultime et du rendement technologique du jambon estimé par la méthode Napole (Mourot et al 1992b).

\section{e / Apport de glycérol}

Du glycérol, coproduit obtenu lors de la fabrication du diester, a été incorporé dans la ration alimentaire de porcs en croissance, en substitution d'environ $5 \%$ de la fraction glucidique. Le glycérol ne modifie ni la vitesse de croissance, ni la composition de la carcasse, mais permet de réduire les pertes en eau du muscle Semi membranosus au ressuyage et lors de la cuisson (Mourot et al 1993). L'effet positif du glycérol sur la capacité de rétention d'eau de la viande a été confirmé par une augmentation du rendement de fabrication du jambon cuit de $2 \%$ chez les animaux supplémentés (Cerneau et al 1994). Ainsi, l'incorporation de glycérol dans la ration alimentaire des porcs apparaît bénéfique du point de vue de la qualité technologique de la viande ; toutefois son utilisation comme matière première par les industries de l'alimentation animale dépend en premier lieu de son coût.

\section{5 / Les promoteurs de croissance}

\section{a / La somatotropine porcine}

L'administration exogène de somatotropine porcine (pST) à des porcs en croissance - finition améliore les performances de croissance (diminution de l'ingéré alimentaire, accélération de la croissance et augmentation de l'efficacité alimentaire) et réduit l'adiposité corporelle au profit du muscle (Bonneau 1992). Les poids des muscles à haute valeur marchande sont augmentés (Lefaucheur et al 1992, Rehfeldt et Ender 1993).

La teneur en lipides intramusculaires est généralement diminuée par le traitement (Lefaucheur et al 1992, Oksbjerg et al 1995), les triglycérides étant seuls affectés (Mourot et al 1992c). En conséquence, le taux d'acides gras polyinsaturés des lipides intramusculaires est augmenté (Mourot et al 1992C, Oksbjerg et al 1995).

Plusieurs études rapportent une hypertrophie de toutes les fibres dans le muscle Longi ssi mus sans modification des pourcentages numériques et des surfaces relatives de chaque type de fibres, suite à l'administration de pST (Lefaucheur et al 1992, Rehfeldt et Ender 1993, Oksbjerg et al 1995).

Concernant la qualité technologique de la viande, l'administration de PST ne modifie généralement ni la vitesse ni l'amplitude de chute du $\mathrm{pH}$ post mortem (Lefaucheur et al 1992, Mourot et al 1992c). Cependant, Oksbjerg et al (1995) rapportent un pH 45 plus bas, alors que Fabry et al (1991) observent un $\mathrm{pH}$ ultime plus élevé. Les mesures physiques de qualité des viandes (tendreté, rétention d'eau et couleur) ne sont généralement pas modifiées par le traitement (Fabry et al 1991, Lefaucheur et al 1992, Oksbjerg et al 1995), alors que Bonneau (1992) et Cannon et al (1995) indiquent dans leur revue que la force de cisaillement et la dureté des viandes sont parfois augmentées. Une analyse sensorielle réalisée par Oksbjerg et al (1995) ne met pas en évidence de différence pour la flaveur la tendreté, la jutosité et l'acceptabilité globale de la viande entre les animaux témoins et traités. L'administration de pST chez le porc en croissance-finition pourrait donc avoir quelques effets négatifs, quoique relativement limités, sur la tendreté des viandes. Cependant, I'utilisation de cette hormone reste actuellement interdite en Europe.

\section{b / Les béta-agonistes}

Les béta-agonistes (ractopamine, salbutamol, cimatérol, clenbutérol) sont des substances pharmaceutiques agonistes des catécholamines. Leur administration à l'animal sain, interdite par la législation actuelle pour la production de viande, permet d'orienter les nutriments vers les tissus maigres au détriment des tissus gras, entraînant une réduction de l'adiposité des carcasses et une augmentation du poids des muscles ( $J$ ones et al 1985, Yen et al 1991).

Le traitement aux béta-agonistes peut entraîner une réduction du taux de lipides intramusculaires ( $J$ ones et al 1985, Yen et al 1991) ; toutefois cet effet n'est pas systématique (Stites et al 1994). Les caractéristiques histologiques des muscles sont modifiées par le traitement. Ainsi, Aalhus et al (1992) montrent que l'administration de ractopamine provoque une augmentation du pourcentage des fibres blanches et du diamètre des fibres blanches et intermédiaires, sans variation de la taille des fibres rouges. Ainsi, les béta-agonistes font évoluer le métabolisme musculaire vers un type plus glycolytique (EssenGustavsson 1993).

La force de cisaillement, la tendreté, la jutosité, la flaveur et la couleur ne sont généralement pas modifiés par l'administration de béta-agonistes aux porcs (Jones et al 1985, Yen et al 1991, Stites et al 1994). Seuls Aalhus et al (1992) signalent une augmentation de la 
force de cisaillement de la viande provenant d'animaux traités. Les effets néfastes marqués de ces produits sur la force de cisaillement et la tendreté des viandes ovines et bovines semblent donc beaucoup moins prononcés chez le porc. Chez les ruminants, I'altération de la tendreté résulterait d'une baisse de l'activité des protéases, conduisant à une diminution de la vitesse et de l'amplitude de la maturation post mortem des viandes. On peut supposer que, chez le porc, l'incidence de ce phénomène sur la tendreté reste relativement limitée, en raison de la vitesse de maturation plus élevée des viandes (Dransfield et al 1981). En conclusion, I'administration de béta-agonistes aux porcs en croissance - finition ne semble pas avoir de conséquences majeures sur les qualités technologiques et sensorielles des viandes. Cependant, l'accumulation possible de résidus dans les tissus reste un problème majeur pouvant affecter la qualité hygiénique des produits.

\section{6 / Les facteurs environnementaux}

\section{a / La température d'élevage}

La température d'élevage influence les besoins énergétiques et les performances de croissance des animaux. La diminution de température accroît la consommation alimentaire spontanée des porcs, qui présentent des besoins d'entretien supérieurs pour assurer leur thermorégulation. Si les animaux sont alimentés à volonté, la diminution de la température ambiante a peu d'incidence sur la composition tissulaire de la carcasse. Par contre, pour un niveau alimentaire donné, les effets d'une faible température sur les propriétés de la carcasse s'apparentent aux effets d'une restriction alimentaire (Le Dividich et al 1998).

La diminution de la température ambiante avec une compensation alimentaire ne modifie pas la teneur en lipides des muscles blancs, alors qu'elle conduit à une augmentation de la teneur en lipides des muscles rouges (Lefaucheur et al 1991 ; tableau 3). La variation de la température ambiante influence le métabolisme musculaire : I'exposition au froid accroît les activités enzymatiques oxydatives musculaires ainsi que le pourcentage et la surface relative des fibres de type I dans les muscles rouges (Dauncey et Ingram 1988, Lefaucheur et al 1991), et augmente la capacité glycolytique (activité LDH) du muscle Longi ssi mus (Lefaucheur et al 1991 ; tableau 3). Une diminution plus faible de la température ambiante $\left(24\right.$ vs $\left.17^{\circ} \mathrm{C}\right)$ ne modifie pas les propriétés histologiques du muscle Longissimus si les porcs sont alimentés à volonté, mais conduit à une augmentation de la surface relative des fibres rouges rapides chez les animaux ne recevant pas de compensation alimentaire (Lebret et al 1998b).

La vitesse et l'amplitude de chute du pH post mortem, ainsi que le potentiel glycolytique du Longissimus sont augmentés chez les porcs élevés à $12^{\circ} \mathrm{C}$ par rapport à $28^{\circ} \mathrm{C}$ (Lefaucheur et al 1991) (tableau 3), alors que l'élevage à $17^{\circ} \mathrm{C}$ comparé à $24^{\circ} \mathrm{C}$ ne modifie pas le pH ultime (Lebret et al 1998b). Une faible température d'élevage pourrait donc avoir des conséquences négatives sur la qualité technologique des muscles blancs. L'influence différentielle des températures basses sur le métabolisme de différents muscles peut également augmenter le phénomène bicolore des viandes (différences de couleur importantes entre deux zones d'une même tranche) et modifier ainsi l'aspect des produits, comme suggéré par Lefaucheur et al (1991) et observé sur des jambons sel - sec issus de porcs élevés à $17^{\circ} \mathrm{C}$, par rapport à d'autres élevés à $24^{\circ} \mathrm{C}$ (Lebret et al 1998b).

\section{b / L'activité physique}

Chez des porcs élevés en système classique, un exercice physique quotidien (marche sur un tapis roulant) influence le métabolisme musculaire. La capacité oxydative (activité $\mathrm{CS}$ ) et le potentiel d'oxydation des lipides (HAD) sont augmentés, et la capacité glycolytique (LDH) ainsi que le rapport de la surface relative des fibres II / I ou $\alpha \mathrm{W} / \alpha \mathrm{R}$ sont parfois diminués dans les muscles Bi ceps femo-
Pour un niveau alimentaire donné, les effets d'une température ambiante basse sont similaires à ceux d'une restriction alimentaire.

Tableau 3. Effet de la température d'élevage sur les caractéristiques musculaires (Lefaucheur et al 1991).

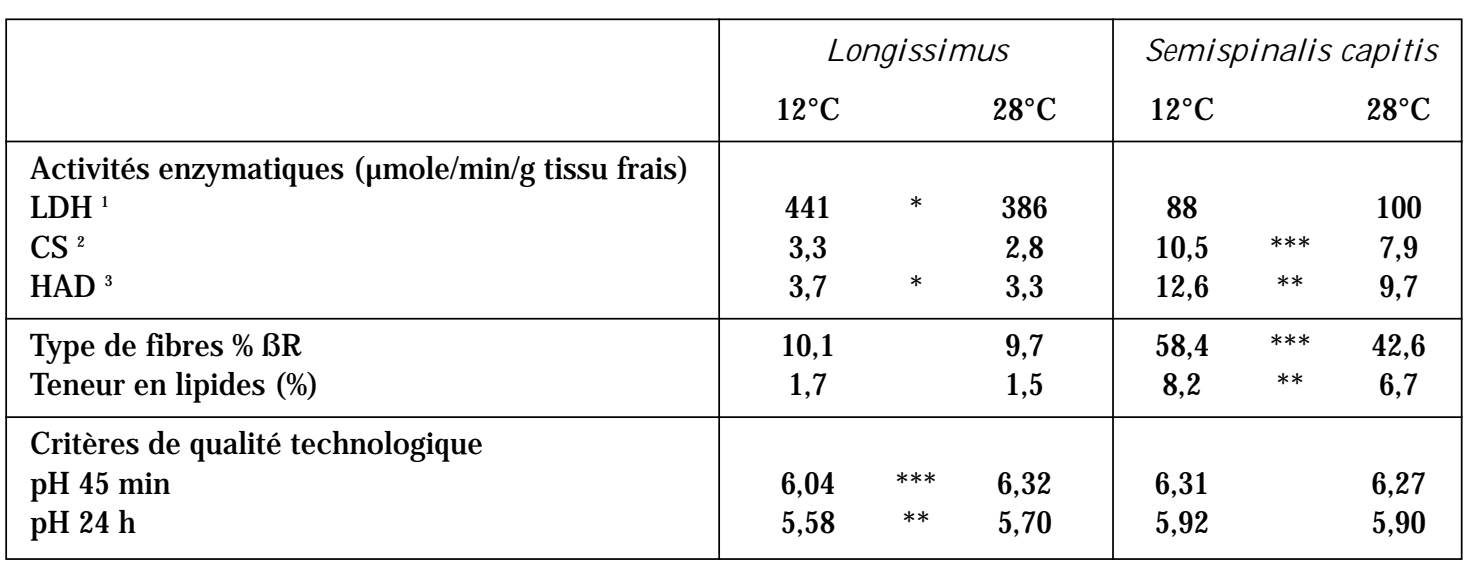

$* \mathrm{P}<0,05 ; * * \mathrm{P}<0,01 ; * * * \mathrm{P}<0,001$.

${ }^{1}$ lactate déshydrogénase; ${ }^{2}$ citrate synthase; ${ }^{3} ß$-hydroxyacyl-coenzyme $A$ déshydrogénase 
ris et Gluteus impliqués dans l'exercice physique (Essen-Gustavsson 1993, Petersen 1997). La composition chimique du muscle Biceps femoris (teneurs en eau, protéines et lipides) et la teneur en collagène intramusculaire ne sont pas modifiées, alors que la teneur en collagène thermostable est augmentée chez les porcs femelles suite à l'exercice (Petersen et al 1997). Aucun de ces effets n'a été observé dans le muscle Longi ssi mus (Petersen 1997, Petersen et al 1997). Ainsi, l'exercice physique semble influencer essentiellement les caractéristiques des muscles directement sollicités par l'exercice. L'augmentation de l'activité physique spontanée des animaux induite par l'accroissement de la surface disponible en élevage pourrait avoir les mêmes effets qu'un exercice régulier sur les caractéristiques des muscles (Petersen et al 1997).

L'activité physique contrôlée ou spontanée en cours d'élevage peut ralentir la vitesse de chute post mortem du $\mathrm{pH}$, le $\mathrm{pH}$ ultime n'étant pas modifié, ou parfois diminué (Petersen 1997). Les mesures mécaniques ou sensorielles de tendreté de la viande cuite ne sont généralement pas influencées par l'activité physique des animaux, malgré une augmentation de la teneur en collagène thermostable et de la force de compression des muscles issus de porcs femelles soumis à une activité physique (Petersen 1997, Petersen et al 1997). Une amélioration de la jutosité du muscle Bi ceps femori s consécutivement à un accroissement de l'activité physique spontanée des porcs a été rapportée ; elle serait attribuée à l'augmentation de la proportion des fibres oxydatives du muscle (Petersen 1997).

\section{c / Le système d'élevage}

Dans le but de promouvoir une meilleure image de marque de la filière porcine auprès des consommateurs et de mieux valoriser les produits, des systèmes d'élevage alternatifs au système intensif classique se mettent en place. Une étude comparant l'élevage en semi plein air (porcs en groupe disposant d'une cabane isolée avec courette extérieure) par rapport à l'élevage en bâtiment fermé à $24^{\circ} \mathrm{C}$, a mis en évidence une augmentation du pourcentage des fibres musculaires rouges rapides $(\alpha R)$ dans le muscle Longissimus chez les porcs en semi plein air (Lebret et al 1998b). Ces auteurs n'observent pas d'influence du mode d'élevage sur la teneur en lipides et le $\mathrm{pH}$ ultime du muscle Semi membranosus. La comparaison d'un système classique d'élevage en intérieur $\left(0,9 \mathrm{~m}^{2} /\right.$ animal $)$ avec un système d'élevage en extérieur ( $\left.50 \mathrm{~m}^{2} / a n i m a l\right)$ n'a pas montré non plus d'effet sur la teneur en lipides, la couleur et le $\mathrm{pH}$ ultime du Longi ssi mus, alors que le $\mathrm{pH}$ ultime et le PRE du Bi ceps femori s étaient diminués en élevage extérieur (Gandemer et al 1990). Une étude suédoise comparant un système d'élevage intérieur classique à un élevage en extérieur de type extensif a également mis en évidence une diminution du $\mathrm{pH}$ ultime liée à une augmentation du potentiel glycolytique chez les animaux élevés en extérieur (Enfält et al 1997).
Des jambons issus des porcs élevés en semi-plein air et en bâtiment ont été transformés en jambons sel - sec. Le système d'élevage ne modifie pas les rendements de transformation, mais influence l'aspect du jambon : la couleur de la fraction maigre est moins homogène pour les jambons issus de l'élevage en semi plein air, la texture et la flaveur des jambons n'étant pas modifiées (Lebret et al 1998b). Dans leur étude, Gandemer et al (1990) n'avaient pas non plus observé d'influence du mode d'élevage sur les qualités sensorielles (tendreté, jutosité, flaveur) de la viande fraîche. A l'inverse, Enfält et al (1997) rapportent une augmentation de la force de cisaillement et une altération de la tendreté de la jutosité et de l'acceptabilité globale de la viande fraîche issue d'animaux élevés en système extérieur extensif.

Selon les études, l'élevage en plein air de porcs charcutiers n'aurait donc pas d'effet, ou des effets plutôt négatifs sur les qualités technologiques et sensorielles de la viande fraîche et des produits transformés. Par ailleurs, les moindres performances de croissance des animaux dues aux variations climatiques engendrent une augmentation des coûts de production, qui doit être compensée par une augmentation de la valeur ajoutée réalisée sur les produits.

\section{Conclusion}

Les caractéristiques des différents constituants musculaires influencent les composantes organoleptique, technologique, nutritionnelle et hygiénique des viandes. Certaines relations sont maintenant bien établies, comme l'effet favorable, jusqu'à $3 \%$ environ, du taux de lipides intramusculaires sur la flaveur et la jutosité. En revanche, les relations entre les caractéristiques des fibres musculaires (type, diamètre, nombre total de fibres) et la qualité ultérieure de la fraction maigre de la viande ne sont pas encore bien identifiées. La teneur en lipides intramusculaires et les propriétés des fibres musculaires peuvent être modifiées sous l'effet de différents facteurs d'élevage. Pour un génotype donné, toute technique d'élevage conduisant à une réduction de l'adiposité globale des carcasses peut se traduire par une diminution plus ou moins importante du taux de lipides intramusculaires, avec des répercussions probables sur la qualité organoleptique des viandes. Les types contractile et métabolique et le diamètre des fibres musculaires sont influencés par certains facteurs environnementaux comme la température ambiante ou l'activité physique des animaux, toutefois les conséquences sur la qualité ne sont pas encore clairement identifiées.

Les demandes socio-économiques en matière de qualité des viandes porcines sont multiples. La première, commune aux consommateurs et aux transformateurs, concerne la qualité hygiénique de la viande et la sécurité alimentaire. Les autres demandes divergent en fonction des utilisateurs. Les transforma- 
teurs recherchent des matières premières de caractéristiques bien connues, homogènes et présentant de bonnes aptitudes à la transformation. Jusqu'à aujourd'hui, la majorité des consommateurs recherchait essentiellement des produits standards, peu chers et présentant des qualités organoleptiques et nutritionnelles satisfaisantes, afin de satisfaire la consommation courante. Actuellement, la demande des consommateurs se diversifie. A côté du besoin en produits standards, on observe une demande croissante pour des produits de qualité organoleptique supérieure (produits festifs), ou pour des produits issus de conditions d'élevage différentes des conditions intensives classiques : productions biologiques, ou types de production respectant le bien-être des animaux et l'environnement.

La satisfaction des demandes variées de la société en matière de qualité des viandes porcines se traduit au niveau de la recherche par la nécessité d'identifier les caractéristiques des tissus maigres favorables aux différentes composantes de la qualité, ainsi que par une meilleure connaissance des mécanismes de différenciation et de développement des tissus et de leurs facteurs de variation, génétiques et d'élevage.

\section{Références}

Aalhus J.L., Schaefer A.L., Murray A.C., J ones S.D.M. 1992. The effect of ractopamine on myofibre distribution and morphology and their relation to meat quality in swine. Meat Sci., 31, 397-409.

Ahn D.U., Lutz S., Sim J S., 1996. Effects of dietary $\alpha$-linolenic acid on the fatty acid composition, storage stability and sensory characteristics of pork loin. Meat Sci., 43 , 291-299.

Ashmore C.R., Doerr L., 1971. Comparative aspects of muscle fibre types in different species. Exp. Neurol., 31, 408-418.

Ashmore C.R., Addis P.B., Doerr L., 1973. Development of muscle in the fetal pig. J. Anim. Sci., 36, 1088-1093.

Bailey A.J ., Light N.D., 1989. Connective tisue in meat and meat products. Elsevier Applied Science, London, 355 p.

Barton-Gade P.A., 1987. Meat and fat quality in boars, castrates and gilts. Livest. Prod. Sci., 16, 187-196.

Bereskin B., Rough D.K., Davey R.J ., 1978. Some factors affecting the evaluation of pork quality. J. Anim. Sci., 47 389-397.

Boccard R.L., Naudé R.T., Cronje D.E., Smit M.C., Venter H.J ., Rossouw E.J., 1979. The influence of age, sex and breed of cattle on their muscle characteristics. Meat Sci. $3,261-280$

Bonneau M., 1988. Intérêts et limites de la production de viandes de porc mâle entier. INRA Prod. Anim., 1, 133-140.

Bonneau M., 1992. Administration de GRF ou de somato tropine chez le porc et les volailles : effets sur les performances, la qualité des viandes et la fonction de reproduction. INRA Prod. Anim., 5, 257-267.

Cameron N.D., Enser M.B., 1991. Fatty acid composition of lipid in Longissimus dorsi muscle of Duroc and British Landrace pigs and its relationship with eating quality. Meat Sci., 29, 295-307.

Candek-Potokar M., Zlender B., Lefaucheur L., Bonneau M., 1998. Effects of age and/or weight at slaughter on longissimus dorsi muscle : biochemical traits and sensory quality in pigs. Meat Sci., 48, 287-300.

Cannon J.E., Morgan J.B. Heavner J., McKeith F.K. Smith G.C." Meeker D.L." 1995. Pork quality audit: a review of the factors influencing pork quality. J. Muscle Foods, 6, 369-402.

Carpenter Z.L., Kauffman R.G., Bray R.W., Briskey E.J ., Weckel K.G., 1963. Factors influencing quality in porks A. Histological observations. J. Food Sci., 28, 467-471.
Cassens R.G., Cooper C.C., 1971. Red and white muscle. Adv. Food Res., 19, 1-74.

Castell A.G., Cliplef R.L., Poste-Flynn L.M., Butler G., 1994. Performance, carcass and pork characteristics of castrates and gilts self-fed diets differing in protein content and lysine:energy ratio. Can. J. Anim. Sci., 74, 519-528.

Cerneau P., Mourot J ., Peyronnet C., 1994. Effet du glycérol alimentaire sur la qualite de la viande de porc et le rendement technologique du jambon cuit. Journées Rech. Porcine en France, 26, 193-198.

Cheah K.S., Cheah A.M., Krausgrill D.I., 1995. Effect on dietary supplementation of vitamin $\mathrm{E}$ on pig meat quality. Meat Sci., 39, 255-264.

Cisneros F., Ellis M., McKeith F.K., McCaw J ., Fernando R.L., 1996a. Influence of slaughter weight on growth and carcass characteristics, commercial cutting and curing yields, and meat quality of barrows and gilts from two genotypes. J. Anim. Sci., 74, 925-933.

Cisneros F., Ellis M., Baker D.H., Easter R.A., McKeith F.K., 1996b. The influence of short-term feeding of amino acid-deficient diets and high dietary leucine levels on the intramuscular fat content of pig muscle. Anim. Sci., 63, 517-522.

Cromwell G.L., Hays V.W., Trujillo-Figueroa V., Kemp J .D., 1978. Effects of dietary protein and energy levels for growing-finishing swine on performance, muscle composition and eating quality of pork. J. Anim. Sci., 47, 505-513.

Dauncey M.J., Ingram D.L., 1988. Influence of environmental temperature and energy intake on skeletal muscle respiratory enzymes and morphology. Eur. J. Appl. Physiol., 58, 239-244.

Desmoulin B., Girard J.P., Bonneau M., Frouin A., 1983. Aptitudes à l'emploi des viandes porcines suivant le type sexuel, le système d'alimentation et le poids d'abattage. J ournées Rech. Porcine en France, 15, 177-192.

De Vol D.L., McKeith F.K., Bechtel P.J ., Novakofski F.K., Shanks R.D Carr TR, 1988. Variation in composition and palatability traits and relationships between muscle characteristics and palatability in a random sample of pork carcasses. J. Anim. Sci., 66, 385-395.

Dirinck $P$, de Winne A., 1995. Effet des taux élevés de supplémentation en vitamine $E$ sur la qualité sensorielle des viandes de porc. J ournées Rech. Porcine en France, 27, 323-328.

Dransfield E., Jones R.C.D., MacFie H.J.H., 1981. Tenderising in M. Longi ssi mus dorsi of beef, veal, rabbit, lamb and pork. Meat Sci., 5, 139-147. 
Eikelenboom G., Hoving-Bolink A.H., Van der Wal P.G., 1996a. The eating quality of pork. 1 . Influence of ultimate pH. Fleischwirtsch., 76, 392-393.

Eikelenboom G., Hoving-Bolink A.H., Van der Wal P.G., $1996 \mathrm{~b}$. The eating quality of pork. 2. The influence of intramuscular fat. Fleischwirtsch., 76, 517-518.

Ellis M., Webb A.J., Avery P.J., Brown I., 1996. The influence of terminal sire genotype, sex, slaughter weight, feeding regime and slaughter-house on growth performance and carcass and meat quality in pigs and on the organoleptic properties of fresh pork. Anim. Sci., 62, $521-530$

Enfält A.C., Lundström K., Hansson I., Lundeheim N., Nyström P.E., 1997. Effects of outdoor rearing and sire breed (Duroc or Yorkshire) on carcass composition and sensory and technological meat quality. Meat Sci., $45,1-15$

Essen-Gustavsson B., 1993. Muscle fiber characteristics in pigs and relationships to meat quality parameters Review. In : E. Puolanne, D.I. Demeyer, M. Ruusunen and S. Ellis (eds), Pork quality: genetic and metabolic factors, 140-159. CAB International, Wallingford, UK.

Essen-Gustavsson B., Fjelkner-Modig S., 1985. Skeletal muscle characteristics in different breeds of pigs in relation to sensory properties of meat. Meat Sci., 13, 33-47.

Essen-Gustavsson B., Karlsson A., Lundström K., Enfält A.C., 1994. Intramuscular fat and muscle lipid contents in halothane-gene-free pigs fed high or low protein diets and its relation to meat quality. Meat Sci., 38, 269-277.

Fabry J., Demeyer D., Thielemans M.F., Deroanne C., Van der Voorde G., Deroover E., Dalrymple R.H., 1991. Evaluation of recombinant porcine somatotropin on growth performance, carcass characteristics, meat quality, and muscle biochemical properties of belgian landrace pigs. J. Anim. Sci., 69, 4007-4018.

Fernandez X., Tornberg E., 1991. A review of the causes of variation in muscle glycogen content and ultimate $\mathrm{pH}$ in pigs. J. Muscle Foods, 2, 209-235.

Fernandez X., Mourot J., Mounier A., Ecolan P., 1995. Effect of muscle type and food deprivation for $24 \mathrm{~h}$ on the composition of the lipidic fraction in muscles of Large White pigs. Meat Sci., 41, 335-343.

Fernandez X., Monin G., Talmant A., Mourot J ., Lebret B., Bernard P., Gilbert S., Sirami J ., Malter D., 1996. Influence de la teneur en lipides intramusculaires sur l'acceptabilité, par les consommateurs, de la viande de porc et du jambon cuit. Journées Rech. Porcine en France, 28, 163-170.

Gandemer G., Pichou D., Bouguennec B., Caritez J.C., Berge P., Briand E., Legault C., 1990. Influence du système d'élevage et du génotype sur la composition chimique et les qualités organoleptiques du muscle Longissimus chez le porc. Journées Rech. Porcine en France, 22, 101-110.

Girard J .P., Bout J ., Salort D., 1988. Lipides et qualités du tissu adipeux, facteurs de variation. Journées Rech. Porcine en France, 20, 255-278.

Goutefongea R., Girard J.P., Jacquet B., 1978. Caractéristiques de la viande de porc et transformation. J ournées Rech. Porcine en France, 10, 235-248.

Hegarty P.V.J ., Allen C.E., 1978. Effect of pre-natal runting on the post-natal development of skeletal muscle in swine and rats. J. Anim. Sci., 46, 1634-1640.

Henry Y., 1993. Alimentation du porc pour la production de viande maigre. Evolutions récentes et perspectives. INRA Prod. Anim., 6, 31-45.

Hill F., 1966. The solubility of intramuscular collagen in meat animals of various ages. J. Food Sci., 31, 161-166.
Hodgson R.R., Davis G.W., Smith G.C., Savell J.W., Cross H.R., 1991. Relationship between pork loin palatability traits and physical characteristics of cooked chops. J. Anim. Sci., 69, 4858-4865.

J ones R.W., Easter R.A., McKeith F.K., Dalrymple R.H., Maddock H.M., Bechtel P.J ., 1985. Effect of the beta-adrenergic agonist cimaterol (CL 263,780) on the growth and carcass characteristics of finishing swine. J. Anim. Sci. $61,905-913$

J oubert D.M., 1956. Analysis of factors influencing postnatal growth and development of the muscle fibre. J. Agric. Sci., 47, 59-102.

Karlsson A., Essen-Gustavsson B., Lundström K., 1992 Muscle glycogen depletion pattern in Longi ssi mus dorsi muscle of pigs fed high and low protein diet. 38th Int Cong. of Meat Sci. Technol., Clermont-Ferrand, France, 3, 375-379.

Klosowska D., Klosowski B., Kortz J ., 1975. Relation between histological picture of musculus Longissimus dorsi and meat quality properties. Proc. of the European Meeting of Meat Research Workers, 21, 73-75.

Laborde D., Talmant A., Monin G., 1985. Activités enzymatiques métaboliques et contractiles de 30 muscles de porc. Relations avec le $\mathrm{pH}$ ultime atteint après la mort. Reprod. Nutr. Develop., 25, 619-628.

Larzul C., Lefaucheur L., E colan P., Gogué J ., Talmant A. Sellier P., Le Roy P., Monin G., 1997. Phenotypic and genetic parameters for Longissimus muscle fiber characteristics in relation to growth, carcass, and meat quality traits in Large White pigs. J. Anim. Sci., 75, 3126-3137.

Lazo A., Gandemer G., Viau M., Rampon V., Gruand J ., Le J ossec P., Chevillon P., 1994. Evolution de la composition lipidique du muscle Longissimus au cours du développement post-sevrage chez trois génotypes porcins. journées Rech. Porcine en France, 26, 175-182.

Lebret B., Mourot J ., 1998. Caractéristiques et qualité des tissus adipeux chez le porc. Facteurs de variation non génétiques. INRA Prod. Anim., 11, 131-143.

Lebret B., Listrat A., Clochefert N., 1998a. Age-related changes in collagen characteristics of porcine loin and ham muscles. 44th Int. Cong. of Meat Sci. Technol., Barcelona, Spain. Paper B111.

Lebret B., Massabie P., J uin H., Mourot J ., Clochefert $\mathrm{N}$. Mounier A., Chevillon P., Bouyssière M., Le Denmat M., 1998b. Influence des conditions d'élevage sur les performances de croissance du porc et la qualité des viandes. 2 Caractéristiques du muscle et du tissu adipeux et qualite technologique et organoleptique du jambon sel-sec. J ournées Rech. Porcine en France, 30, 43-50.

Le Dividich J ., Noblet J ., Herpin P., Van Milgen J ., Quiniou N., 1998. Thermoregulation. In : J . Wiseman, M.A. Varley and J.P. Chadwick (eds), Progress in pig science, 229-263. Nottingham University Press, Nottingham, UK.

Lee Y.B., Kauffman R.G., 1974. Cellular and enzymatic changes with animal growth in porcine intramuscular adipose tissue. J. Anim. Sci., 38, 532-537.

Lee Y.B., Kauffman R.G., Grummer R.H., 1973a. Effect of early nutrition on the development of adipose tissue in the pig. I. Age constant basis. J. Anim. Sci., 37, 1312-1318.

Lee Y.B., Kauffman R.G., Grummer R.H., 1973b. Effect of early nutrition on the development of adipose tissue in the pig. II. Weight constant basis. I. Anim. Sci., 37, 1319-1325.

Lefaucheur L., 1983. Influence d'une restriction alimentaire sur la composition de la carcasse et quelques carac téristiques musculaires chez le porc. DEA, Université Clermont II, $41 \mathrm{p}$. 
Lefaucheur L., 1989. Les différents types de fibres musculaires chez le porc. Conséquences sur la production de viande. INRA Prod. Anim., 2, 205-213.

Lefaucheur L., Vigneron P., 1986. Post-natal changes in some histochemical and enzymatic characteristics of three pig muscles. Meat Sci., 16, 199-216.

Lefaucheur L., Le Dividich J ., Mourot J ., Monin G., E colan P., Krauss D., 1991. Influence of environmental temperature on growth, muscle and adipose tissue metabolism, and meat quality in swine. J. Anim. Sci., 69, 2844-2854.

Lefaucheur L., Missohou A., Ecolan P., Monin G., Bonneau M., 1992. Performance, plasma hormones, histochemical and biochemical muscle traits, and meat quality of pigs administered exogenous somatotropin between 30 or 60 and 100 kilograms body weight. J. Anim. Sci., 70, 3401-3411.

Lefaucheur L., Edom F., Ecolan P., Butler-Browne G.S 1995. Pattern of muscle fiber type formation in the pig. Develop. Dynamics, 203, 27-41.

Lefaucheur L., Hoffman R., Okamura C., Gerrard D. Léger J.J., Rubinstein N., Kelly A., 1997. Transitory expression of alfa cardiac myosin heavy chain in a subpopulation of secondary generation muscle fibers in the pig. Develop. Dynamics, 210, 106-116.

Lefaucheur L., Hoffman R., Gerrard D., Okamura C. Rubinstein N., Kelly A., 1998. Evidence of three adult fast myosin heavy chain isoforms in type II skeletal muscle fibers in pigs. J. Anim. Sci., 76, 1584-1593.

Leseigneur-Meynier A., Gandemer G., 1991. Lipid composition of pork muscle in relation to the metabolic type of the fibers. Meat Sci., 29, 229-241.

Livingston D.M.S., Blair R., English P.R., 1966. The usefulness of muscle fiber diameter in studies of the lean meat and content of pigs. Anim. Prod., 8, 267-274.

Malmfors B., Lundström K., Hansson I., 1978. Fatty acid composition of porcine back fat and muscle lipids as affected by sex, weight and anatomical location. Swedish J. Agric. Res., 8, 25-38.

Miller L.R., Garwood V.A., J udge M.D., 1975. Factors affecting porcine muscle fiber type, diameter and number. J. Anim. Sci., 41, 66-77.

Minelli G., Culioli ] ., Vignon X., Monin G., 1995. Post mortem changes in the mechanical properties and ultrastruc ture of the Longi ssi mus in two porcine breeds. J. Muscle Foods, 6, 313-326.

Monahan F.J., Buckley D.J., Gray J.I., Morissey P.A Asghar A., Hanrahan T.J ., Lynch P.B., 1990. Effect of dietary vitamin $\mathrm{E}$ on the stability of raw and cooked pork. Meat Sci., 27, 99-108.

Monahan F.J., Asghar A., Gray J.I., Buckley D.J. Morrissey P.A., 1992. Influence of dietary vitamine E ( $\alpha$ tocopherol) on the colour stability of pork chops. 38th Int. Cong. of Meat Sci. Technol., Clermont-Ferrand France, 3, 543-546.

Monin G., 1983. Influence des conditions de production et d'abattage sur les qualités technologiques et organoleptiques des viandes de porc. J ournées Rech. Porcine en France, 15, 151-176.

Monin G., 1988. Evolution post mortem du tissu musculaire et conséquences sur les qualités de la viande de porc. J ournées Rech. Porcine en France, 20, 201-214.

Monin G., Talmant A., Laborde D., Zabarri M., Sellier P., 1986. Compositional and enzymatic characteristics of the Longi ssi mus dorsi muscle from Large White, halothanepositive and halothane-negative Pietrain, and Hampshire pigs. Meat Sci., 16, 307-316.
Monin G., Mejenes-Quijano A., Talmant A., Sellier P., 1987. Influence of breed and muscle metabolic type on muscle glycolytic potential and meat $\mathrm{pH}$ in pigs. Meat Sci., 20, 149-158.

Monin G., Larzul C., Le Roy P., Culioli J., Mourot J., Rousset-Akrim S., Talmant A., Sellier P., 1999. Effects of the halothane genotype and slaughter weight on texture of pork. J. Anim. Sci., sous presse.

Mottram D.S., Edwards R.A., 1983. The role of triglycerides and phospholipids in the aroma of cooked beef. J. Sci. Food Agric., 34, 517-522.

Mourot J., Kouba M., 1995. Development of intramuscular adipose tissue and effects on meat quality in growing Large White and Meishan pigs. 2nd Dummerstorf MuscleWorkshop "Muscle Growth and Meat Quality", Rostock, Germany, 133-140.

Mourot J ., Chauvel J ., Le Denmat M., Mounier A., Peiniau P., 1991. Variations du taux d'acide linoléique dans le régime du porc : effets sur les dépôts adipeux et sur l'oxydation du C18:2 au cours de la conservation de la viande. J ournées Rech. Porcine en France, 23, 357-364.

Mourot J., Aumaitre A., Mounier A., 1992a. Interaction entre vitamine $E$ et acide linoléique alimentaires : effet sur la composition de la carcasse, la qualité et la conservation des lipides de la viande chez le porc. Sciences des Aliments, 12, 743-755.

Mourot J., Aumaitre A., Peiniau P., Chevillon P., 1992b. Effet de l'apport de vitamine $C$ sur les performances de croissance et la qualité de la viande chez des porcs Large White et croisés Large White Piétrain. Journées Rech. Porcine en France, 24, 55-64.

Mourot J., Bonneau M., Charlotin P., Lefaucheur L., 1992c. Effects of exogenous somatotropin (pST) administration on pork meat quality. Meat Sci., 31, 219-227.

Mourot J ., Aumaitre A., Mounier A., Peiniau P., François A., Peyronnet C., J amet J.-P., 1993. Effets du glycérol alimentaire sur les performances de croissance et la qualité de la viande chez le porc Large White. J ournées Rech. Porcine en France, 25, 29-36.

Mourot J ., Kouba M., Peiniau P., 1995. Comparative study of in vitro lipogenesis in various adipose tissues in the growing pig (Sus domesti cus). Comp. Biochem. Physiol., 111B, 379-384.

Offer G., Knight P., J eacocke R., Almond R., Cousins T., Elsey J., Parsons N., Sharp A., Starr R., Purslow P., 1989. The structural basis of the water-holding, appearance and toughness of meat and meat products. Food Microstr., 8 , 151-170.

O'Halloran G.R., Troy D.J., Buckley D.J., Reville W.J., 1997. The role of endogenous proteases in the tenderisation of fast glycolysing muscle. Meat Sci., 47, 187-210.

Oksbjerg N., Petersen J.S., Sorensen M.T., Henckel P., Agergaard N., Bejerholm C., Erlandsen E., 1995. The influence of porcine growth hormone on muscle fibre characteristics, metabolic potential and meat quality. Meat Sci., 39, 375-385.

Ouali A., 1991. Conséquences des traitements technologiques sur la qualité de la viande. INRA Prod. Anim., 4, 195-208.

Ouali A., Talmant A., 1990. Calpains and calpastatin distribution in bovine, porcine and ovine skeletal muscles. Meat Sci., 28, 331-348.

Petersen J.S., 1997. Muscle structure and meat quality in physically active pigs. Pig News Inf., 18, 79N-82N .

Petersen J S., Berge P., Henckel P., Maribo H., Oksbjerg N. Sorensen M.T., 1994. Muscle structure and texture as influenced by physical exercise of pigs. 40th Int. Cong. of Meat Sci. Technol., The Hague, Netherlands, paper S-IVA. 
Petersen J.S., Berge P., Henckel P., Sorensen M.T., 1997. Collagen characteristics and meat texture of pigs exposed to different levels of physical activity. J. Muscle Foods, 8, 47-61.

Powell S.E., Aberle E.D., 1980. Effects of birth weight on growth and carcass composition of swine. J. Anim. Sci., $50,860-868$

Ramsey C.B., Tribble L.F., Wu C., Lind K.D., 1990. Effect of grains, marbling and sex on pork carcass tenderness and composition. J. Anim. Sci., 68, 148-154.

Rehfeldt C., Ender K., 1993. Skeletal muscle cellularity and histochemistry in response to porcine somatotropin in finishing pigs. Meat Sci., 34, 107-118.

Renard C., Mourot J ., Götz K.U., Caritez J .C., Bidanel J .P., Vaiman M., 1992. Analyse des liaisons génétiques entre le marqueurs SLA et les caractères de croissance et d'adiposité chez le porc. Journées Rech. Porcine en France, 24, 9-16

Renou J.P., Monin G., Sellier P., 1985. Nuclear magnetic resonance measurements on pork of various qualities. Meat Sci., 15, 225-233.

Sellier P., 1988. Aspects génétiques des qualités technologiques et organoleptiques de la viande chez le porc. Journées Rech. Porcine en France, 20, 227-242.

Sellier P., 1998. Genetics of meat and carcass traits. In : M.F. Rotschild and A. Ruvinsky (eds), The Genetics of the pig, 463-510. CAB International, Wallingford, UK.

Sellier P., Monin G., 1994. Genetics of pig meat quality. J . Muscle Foods, 5, 187-219.

Staun H., 1963. Various factors affecting number and size of muscle fibers in the pig. Acta Agric. Scand., 13, 292-322.

Stites C.R., McKeith F.K., Singh S.D., Bechtel P.J ., J ones D.J., Mowrey D.H., 1994. Palatability and visual characteristics of hams and loin chops from swine treated with ractopamine hydrochloride. J. Muscle Foods, 5, 367-376.

Talmant A., Monin G., Briand M., Dadet M., Briand Y., 1986. Activities of metabolic and contractile enzymes in 18 bovine muscles. Meat Sci., 18, 23-40.
Tarrant P.V., 1998. Some recent advances and future priorities in research for the meat industry. Meat Sci., 49, S1-S16.

Touraille C., Monin G., Legault C., 1989. Eating quality of meat from european $X$ chinese crossbred pigs. Meat Sci. $25,177-186$

Valin C., 1988. Différenciation du tissu musculaire Conséquences technologiques pour la filière viande. Reprod. Nutr. Develop., 28, 845-856.

Valin C., Touraille C., Vigneron P., Ashmore C.R., 1982. Prediction of lamb meat quality traits based on muscle biopsy fibre typing. Meat Sci., 6, 257-263.

Van Oeckel M.J., Casteels M., Warnants N., Van Damme L., Boucqué C.V., 1996. Omega-3 fatty acids in pig nutrition: implications for the intrinsic and sensory quality of the meat. Meat Sci., 44, 55-63.

Wigmore P.M.C., Stickland N.C., 1983. Muscle development in large and small pig fetuses. J. Anat., 137, 235-245.

Wood J .D., Enser M., 1996. Factors influencing fatty acids in meat and the role of antioxidants in improving meat quality. International conference "Fats in the diet of animals and man", Birmingham, UK, 4 p.

Wood J.D., Jones R.C.D., Francombe M.A., Whelehan O.P., 1986. The effects of fat thickness and sex on pig meat quality with special reference to the problems associated with overleanness. 2- Laboratory and trained taste panel results. Anim. Prod., 43, 544

Wood J.D., Brown S.N., Nute G.R., Whittington F.M., Perry A.M., J ohnson S.P., Enser M., 1996. Effects of breed, feed level and conditioning time on the tenderness of pork. Meat Sci., 44, 105-112.

Yen J .T., Nienaber J .A., Klindt J ., Crouse J .D., 1991. Effect of ractopamine on growth, carcass traits, and fasting heat production of U.S. contemporary crossbred and chinese Meishan pure-and crossbred pigs. J. Anim. Sci., 69, 4810-4822.

Zamora F., Debiton E., Lepetit J ., Lebert A., Dransfield E., Ouali A., 1996.Predicting variability of ageing and toughness in beef $M$. longi ssi mus lumborum et thoracis. Meat Sci., 43, 321-333.

\section{Abstract}

Pig meat quality. Influence of rearing factors on skeletal muscle traits

Pig meat quality is influenced by interactions between genotype, rearing factors and preslaughter and slaughter conditions. Genotype, particularly the presence of the major genes $\mathrm{Hal}$ and RN-, and slaughter conditions greatly influence meat quality. Once influences of genotype and slaughter conditions are controlled, further improvement of pig meat quality implies to take into account the effects of rearing factors on muscle characteristics and meat quality, which is the objective of this review.

This paper describes muscle characteristics, in particular muscle fibers, intramuscular fat and connective tissue and their relationships with sensory, technological, nutritional and hygienic traits of meat. Thereafter, influences of rearing factors : age and weight at slaughter, sex, feeding level, diet composition, growth promo- tors, ambient temperature, exercise and rearing conditions on muscular tissue traits and meat quality are described. Although some relationships are well established, such as the favorable effect of intramuscular fat level up to $3 \%$ on sensory properties, the influence of muscle fiber characteristics (metabolic and contractile type, diameter) on the different components of meat quality is not fully understood. The identification of muscle traits that are favorable to meat quality, and a better knowledge of the mechanisms leading to the differentiation and development of muscular tissues are required, in order to produce meat that satisfies consumers as well as the meat industry.

Lebret B., Lefaucheur L., Mourot J ., 1999. La qualité de la viande de porc. Influence des facteurs d'élevage non génétiques sur les caractéristiques du tissu musculaire. INRA Prod. Anim., 12, 11-28. 